\title{
Diophantine Representation in Thin Sequences
}

\author{
Dissertation \\ zur Erlangung des mathematisch-naturwissenschaftlichen Doktorgrades \\ "Doctor rerum naturalium" \\ der Georg-August-Universität Göttingen \\ im Promotionsprogramm (SMS) \\ der Georg-August-Universität Göttingen (GAUSS)
}

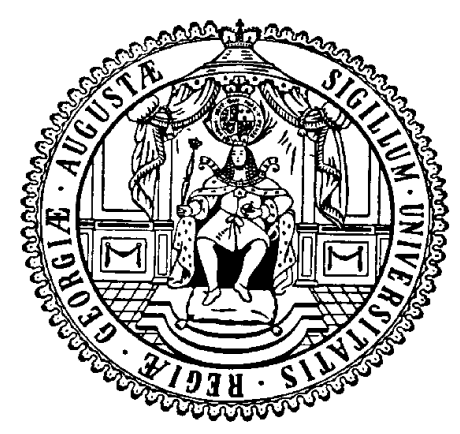

vorgelegt von

Stefan Baur

aus Stuttgart

Göttingen, 2016 


\section{Betreuungsausschuss}

Prof. Dr. Jörg Brüdern, Mathematisches Institut Göttingen

Prof. Dr. Valentin Blomer, Mathematisches Institut Göttingen

\section{Mitglieder der Prüfungskommission}

Referent: Prof. Dr. Jörg Brüdern, Mathematisches Institut Göttingen

Koreferent: Prof. Dr. Valentin Blomer, Mathematisches Institut Göttingen

Weitere Mitglieder der Prüfungskommission

PD Dr. Ulf-Rainer Fiebig, Institut für Mathematische Stochastik Göttingen

Prof. Dr. Ina Kersten, Mathematisches Institut Göttingen

Prof. Dr. Russell Luke, Institut für Numerische und Angewandte Mathematik

Prof. Dr. Preda Mihăilescu, Mathematisches Institut Göttingen

Tag der mündlichen Prüfung: 21. April 2016 


\section{Contents}

1 Overview 1

1.1 Notation . . . . . . . . . . . . . . . . . . 5

2 General Method $\quad 6$

3 Forms of degree $d \quad 10$

3.1 Minor Arcs . . . . . . . . . . . . . . . . . . . . . 11

3.2 Major Arcs . . . . . . . . . . . . . . . . . . . . 12

3.2.1 Singular Series . . . . . . . . . . . . . . . . 12

3.2 .2 Singular Integral _. . . . . . . . . . . . . . . 15

3.3 Conclusion . . . . . . . . . . . . . . . . . . . 16

4 Cubic Forms $\quad 17$

4.1 Preparations . . . . . . . . . . . . . . . . . . . . . . 18

4.2 Major Arcs . . . . . . . . . . . . . . . . . . . . . . . . . . . . . . . . . . . . . . .

4.3 Minor Arcs . . . . . . . . . . . . . . . . . . . . . 23

4.4 Conclusion . . . . . . . . . . . . . . . . . . . . . . 31

5 Quartic Forms 33

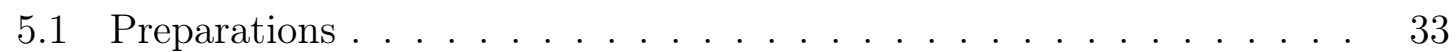

5.2 Major Arcs . . . . . . . . . . . . . . . . . . . . . . . 33

5.2 .1 Singular Series . . . . . . . . . . . . . . . 35

5.2 .2 Singular Integral f . . . . . . . . . . . . . . . . 36

5.3 Minor Arcs . . . . . . . . . . . . . . . . . . . . . 37

5.4 Conclusion . . . . . . . . . . . . . . . . . . . . . . . . 39

6 Conclusion $\quad 40$

$\begin{array}{ll}\text { Bibliography } & 43\end{array}$ 



\section{Overview}

In 1770 Waring proposed the problem, whether each natural number $n$ can be written as the sum of $k$-th powers of natural numbers, as a generalization of an old problem of the ancient greek Diophantus of Alexandria, who asked whether each natural number $n$ can be written as the sum of four integer squares. Waring's question has become famous as the so-called Waring's problem and Hilbert [16] managed to prove, that indeed, for every $k$ there is a finite number $s$ such that every $n$ is the sum of at most $s k$-th powers of natural numbers. This problem inspired many authors to come up with new methods to further reduce the number of variables needed and lead to many generalizations in the structure of the underlying diophantine equation.

In this work we want to replace the sum of $k$-th powers by homogeneous polynomials, more precisely let $f \in \mathbb{Z}\left[x_{1}, \ldots, x_{s}\right]$ be a form of degree $d$. We say that $f$ represents $n \in \mathbb{N}$, if the diophantine equation $f(\mathbf{x})=n$ has integral solutions $\mathbf{x}=\left(x_{1}, \ldots, x_{s}\right) \in$ $\mathbb{Z}^{s}$. As with fewer variables it gets more unlikely to have solutions to the equation $f(\mathbf{x})=n$, one can ask for good bounds on $s$ in order to guarantee that every $n \in \mathbb{N}$ is represented by $f$. A variant of this problem has been studied by Birch [1], who was mainly interested in the special case $n=0$ and also considered systems of equations.

A further generalization is to allow (few) exceptional $n$, which might not be represented by $f$. Thus one can ask whether almost all $n \in \mathcal{A}$ can be represented by the form $f$, for a (infinite) subset $\mathcal{A} \subset \mathbb{N}$. Or more precisely, if we denote the set of exceptional $n$ up to $N$ by

$$
\mathcal{E}_{N}:=\{n \in \mathcal{A}: n \leq N, f \text { does not represent } n\},
$$

then the goal will be to show that

$$
\lim _{N \rightarrow \infty} \frac{\left|\mathcal{E}_{N}\right|}{|\mathcal{A} \cap\{1,2, \ldots N\}|}=0
$$

This will be done using the circle method to transform the problem into a problem of estimating certain exponential sums.

For $\mathcal{A}=\mathbb{N}$, a rather classical approach using Bessel's inequality would lead to the problem of finding appropriate upper bounds for the minor arc contribution to the diophantine problem $f(\mathbf{x})=f(\mathbf{y})$ in the $2 s$ variables $(\mathbf{x}, \mathbf{y}) \in \mathbb{Z}^{2 s}$. So roughly 
speaking, one can hope that only half as many variables are needed to give a positive answer, as would be for the corresponding problem of finding non-trivial solutions of $g(\mathbf{x}, \mathbf{y})=0$, where $g(\mathbf{x}, \mathbf{y})=f(\mathbf{x})-f(\mathbf{y})$. Thus one saves half the number of variables by asking for almost all $n \in \mathbb{N}$ to be represented.

In our case we want to give similar answers when $\mathcal{A}$ is a thin (but infinite) set, namely for $\mathcal{A}_{N}:=\mathcal{A} \cap\{1,2, \ldots N\}$, we require $\lim _{N \rightarrow \infty} N^{-\frac{1}{k}}\left|\mathcal{A}_{N}\right|=c>0$ for some $k \geq 2$.

Especially we consider the case of $\mathcal{A}=\{\mathfrak{q}(m): m \in \mathbb{N}\} \cap \mathbb{N}$, the positive values of an integral quadratic polynomial $\mathfrak{q}$ with positive leading coefficient, e.g. the set of all squares.

Note that this is indeed a harder problem and it is not enough to show that almost all $n \in \mathbb{N}$ are represented by $f$, since then the thin set $\mathcal{A}$ could still be completely contained in the exceptional set. Instead a more flexible way of applying the circle method has to be used. This is where we build on ideas of a series of papers of Brüdern, Kawada and Wooley [5] - [12], concerning additive representations in thin sequences.

Similar to our problem, the authors investigate Waring's problem for cubes in [5] and provide the strong bound $\mathcal{E}_{N} \ll N^{19 / 56}$ for the exceptional set in the case of six cubes representing the positive values of a quadratic polynomial $\mathfrak{q}$ as above and $\mathcal{E}_{N} \ll N^{255 / 548}$ for representing the values of corresponding cubic polynomials. In [7] they show that the expected asymptotic formula for the number of representation holds for almost all values of $\mathfrak{q}$. In the other parts of the series further generalizations are considered, such as replacing Waring's sum of $k$-th powers by Goldbach's problem of sums of two primes in [6] or replacing $\mathcal{A}$ by the set of primes or almost primes in [10].

This emphasizes the strength of this approach. The framework of the general method will be laid out in chapter 2 , adapted to our case of a polynomial $f$ instead of $k$-th powers.

The main task will be to estimate the size of the exceptional set by a minor arc integral, where the number of variables can be thought of being $\frac{4}{3} s$. Such that we will roughly only need $\frac{3}{4}$ as many variables, as for the corresponding problem of $f(\mathbf{x})=0$.

In chapter 3 we will deal with the case of $f$ being a form of arbitrary degree $d$. More precisely, we show

Theorem 1.1. Let $f \in \mathbb{Z}\left[x_{1}, \ldots, x_{s}\right]$ be a form of degree $d$ with singular locus sing $f$ and $\mathcal{A}=\{\mathfrak{q}(m): m \in \mathbb{N}\} \cap \mathbb{N}$ as above. If $s-\operatorname{dim} \operatorname{sing} f>\frac{3}{4} 2^{d}(d-1)$ then almost all $n \in \mathcal{A}$ are represented by $f$, as long as there are no real or p-adic obstructions. 
Where the condition $s-\operatorname{dim} \operatorname{sing} f>\frac{3}{4} 2^{d}(d-1)$ is exactly $\frac{3}{4}$ times the number of variables needed to ensure the solvability of $f(\mathbf{x})=0$ (or $f(\mathbf{x})=n)$ in [1] and almost all $n \in \mathcal{A}$ means $\lim _{N \rightarrow \infty} \frac{\left|\mathcal{E}_{N}\right|}{\left|\mathcal{A}_{N}\right|}=0$.

Note. It is important to ensure that there are no real or p-adic obstructions, as the following simple example illustrates. Let $g \in \mathbb{Z}\left[x_{1}, \ldots, x_{s}\right]$ be an arbitrary polynomial. Take $f(\mathbf{x})=2 g(\mathbf{x})$ and $\mathfrak{q}(m)=2 m^{2}+1$. Then there are no solutions to the equation $f(\mathbf{x})=\mathfrak{q}(m)$ at all, because this would imply $0 \equiv 1 \bmod 2$, so we have a 2-adic obstruction for all $n$.

Similarly, if $f$ is negative definite, it will not be possible to represent positive $n$ for $\mathbf{x} \in \mathbb{R}$ and we have a real obstruction.

As we will see at the end of section 3.2.1, p-adic obstructions lead to certain arithmetic progressions, which are "forbidden" for $n$, together with the complementary progressions containing "good" $n$. So if there is at least one $n \in \mathcal{A}$ without $p$-obstructions, we immediately have infinitely many without obstructions, lying the in the corresponding arithmetic progression of $n$. If we now restrict the set $\mathcal{A}$ to those "good" $n$, a positive proportion of $n \in \mathcal{A}$ will remain and the new set will still be of the same order of magnitude as the original set $\mathcal{A}$. For the proofs of the Theorems, it will be convenient to do so and assume, that $f$ and the set $\mathcal{A}$ are such that $\mathcal{A}$ consists only of those $n$ without real or $p$-adic obstructions for $f$.

Another interpretation of our results with restriction to $n$ without obstructions is, that for almost all $n \in \mathcal{A}$ the Hasse principle holds, meaning that the solvability of $f(\mathbf{x})=n$ in $\mathbb{Z}$ can be decided by checking the solvability in $\mathbb{R}$ and modulo all prime powers $p^{k}$, since for the $n$ with obstructions the Hasse principle holds trivially.

For forms of small degree, more refined results are available than the ones used for arbitrary degree $d$. Namely for $d=3$ Heath-Brown [15] investigated non-singular cubic forms in 10 variables representing 0 and Hooley [17] refined the analysis to 9 variables. We can feed those results into the general method described in chapter 2 to get

Theorem 1.2. Let $f \in \mathbb{Z}\left[x_{1}, \ldots, x_{s}\right]$ be a non-singular cubic form and $\mathcal{A}=\{\mathfrak{q}(m)$ : $m \in \mathbb{N}\} \cap \mathbb{N}$ as above. If $s \geq 8$ then almost all $n \in \mathcal{A}$ are represented by $f$, as long as there are no p-adic obstructions.

This will be done in chapter 4 . Note that in this case there are no real obstructions since $f$ is of odd degree. Unfortunately, not all of Hooley's refinements are directly applicable to our situation. So our method only works for at least 8 , instead of 7 variables, which one might hope for by the heuristic, that our method roughly 
produces results in $\frac{3}{4}$ times the number of variables needed for the corresponding problem $f(\mathbf{x})=0$. See chapter 4.4 for more details.

In chapter 5 we consider forms of degree $d=4$ and use results from Browning and Heath-Brown [4] to deduce

Theorem 1.3. Let $f \in \mathbb{Z}\left[x_{1}, \ldots, x_{s}\right]$ be an absolutely irreducible quartic form defining a quartic hypersurface $X \subset \mathbb{P}_{\mathbb{Q}}^{s-1}$ and $\sigma=\operatorname{dim} \operatorname{sing} X$ the dimension of its singular locus. Further, let $\mathcal{A}=\{\mathfrak{q}(m): m \in \mathbb{N}\} \cap \mathbb{N}$ as above. If $s-\sigma \geq 32$ then almost all $n \in \mathcal{A}$ are represented by $f$, as long as there are no real or $p$-adic obstructions.

Note that Browning and Heath-Brown need $s-\sigma \geq 42$ in order to prove that the Hasse principle holds for $f(\mathbf{x})=0$ and the 32 in Theorem 1.3 is $\frac{4}{3}$ times that number, rounded to the next integer, so in this case we are able to carry the full savings through our method.

We close with a discussion of the general case of sets of size $\left|\mathcal{A}_{N}\right| \sim c N^{\frac{1}{k}}, k \geq 2$ in chapter 6 and give a heuristic for the number of variables one would typically expect to come out of our method. 


\subsection{Notation}

Throughout this work, $N$ will be a cut-off parameter for the set $\mathcal{A}$ and should be thought of large and tending to infinity. We will be searching for solutions in a box of size $B$, which (together with some other parameters) will grow as $N \rightarrow \infty$.

We use the usual Landau O-notation and Vinogradov's « notation to mean "less than a constant times". In this context $\varepsilon$ denotes a number that may be chosen arbitrarily small (and may change on each occurrence). The constants hidden in the O-notation may depend on $\varepsilon$ and the polynomial $f$ under consideration.

We denote a sum over elements $a$, co-prime to $q$ by $\sum_{a=1}^{q}{ }^{*}$, and similarly the union over elements $a$, co-prime to $q$ by $\bigcup_{a=1}^{q} *$. A sum over a complete set $x=1, \ldots, q$ of residue classes modulo $q$ will be denoted by $\sum_{x(q)}$.

The variable $p$ will usually denote a prime number and $p^{\nu} \| q$ states that $\nu$ is the exact power of $p$ dividing $q$. The greatest common divisor of two integers $a$ and $b$ will be denoted by $(a ; b)$.

A sum over elements of size $c_{1} R<q \leq c_{2} R$, for some constants $c_{1}$ and $c_{2}$, will be denoted by $\sum_{q \sim R}$. Usually, if not otherwise noted, the constants can be thought of as being $c_{1}=1$ and $c_{2}=2$.

Elements $\mathbf{x}=\left(x_{1}, \ldots, x_{s}\right) \in \mathbb{Z}^{s}$ are printed bold and summation conditions for such elements should be read component-wise as in $\sum_{|\mathbf{x}| \leq M}=\sum_{\left|x_{1}\right| \leq M} \sum_{\left|x_{2}\right| \leq M} \ldots \sum_{\left|x_{s}\right| \leq M}$.

As usual when working with exponential sums in this context, e $(\alpha)$ will denote the exponential $e^{2 \pi i \alpha}$. 


\section{General Method}

As mentioned in the introduction, we want to transform the problem of deciding, whether the equation $f(\mathbf{x})=n$ has solutions or not, into a problem of finding appropriate bounds on certain exponential sums. This will be done using the HardyLittlewood circle method.

First, instead of searching for arbitrary solutions $\mathbf{x} \in \mathbb{Z}^{s}$, we want to focus on those $\mathbf{x}$ that are somewhat close to a fixed point $\mathbf{x}_{0} \in \mathbb{R}^{s}$. For technical reasons, it will be helpful to do so by introducing a weight as follows.

Let $\omega$ be a suitable weight function with support in $(-1,1)$ and define

$$
\mathrm{w}(\mathbf{x}):=\prod_{j=1}^{s} \omega\left(\rho^{-1}\left(\mathbf{x}-\mathbf{x}_{0}\right)_{j}\right) \text { and } \mathrm{w}_{B}(\mathbf{x}):=\mathrm{w}\left(\mathbf{x} B^{-1}\right),
$$

where $\rho \in(0,1)$ will be taken to be suitably small.

Now define the usual weighted exponential sum

$$
S(\alpha):=\sum_{\mathbf{x} \in \mathbb{Z}^{s}} \mathrm{e}(\alpha f(\mathbf{x})) \mathrm{w}_{B}(\mathbf{x})
$$

By orthogonality we get

$$
\int_{0}^{1} S(\alpha) \mathrm{e}(-\alpha n) d \alpha=\sum_{\mathbf{x} \in \mathbb{Z}^{s}: f(\mathbf{x})=n} \mathrm{w}_{B}(\mathbf{x}),
$$

counting the solutions $\mathbf{x} \in \mathbb{Z}^{s}$ to $f(\mathbf{x})=n$, weighted by $\mathrm{w}$, in a box growing as $B \rightarrow \infty$.

To be able to represent $n \sim N$ by a form of degree $d$, it is a natural choice to search in a box of size $B$, where $B^{d} \sim N$, which we assume from now on. For $n$ in the exceptional set $\mathcal{E}$, there are no solutions to $f(\mathbf{x})=n$, so the right-hand side in (2.3) will be zero.

If we dissect the unit interval into two disjoint sets $[0,1)=\mathfrak{M} \cup \mathfrak{m}$, we obtain

$$
\int_{\mathfrak{M}} S(\alpha) \mathrm{e}(-\alpha n) d \alpha=-\int_{\mathfrak{m}} S(\alpha) \mathrm{e}(-\alpha n) d \alpha
$$


Summing this up over $n \in \mathcal{E}_{N}:=[N, \tau N] \cap \mathcal{E}$, with $\tau>1$, leads to

$$
\sum_{n \in \mathcal{E}_{N}} \int_{\mathfrak{M}} S(\alpha) \mathrm{e}(-\alpha n) d \alpha=-\int_{\mathfrak{m}} S(\alpha) K(\alpha) d \alpha
$$

where $K(\alpha)=\sum_{n \in \mathcal{E}_{N}} \mathrm{e}(-\alpha n)$.

One part of the further investigation will be to prove

$$
\int_{\mathfrak{M}} S(\alpha) \mathrm{e}(-\alpha n) d \alpha \gg B^{s-d-\varepsilon}
$$

for a suitable choice of major arcs $\mathfrak{M}$ and almost all $n \in \mathcal{A}$.

Inserting this into (2.4), we can bound the size of the exceptional set by

$$
\left|\mathcal{E}_{N}\right| \ll B^{-s+d+\varepsilon} \int_{\mathfrak{m}}|S(\alpha) K(\alpha)| d \alpha
$$

Note. At this stage we could apply the Cauchy-Schwarz inequality to recover estimates in the case $\mathcal{A}=\mathbb{N}$ as mentioned in the introduction.

Instead, we continue by applying Hölder's inequality to the minor arc integral in (2.6) to get

$$
\int_{\mathfrak{m}}|S(\alpha)||K(\alpha)| d \alpha \ll\left(\int_{\mathfrak{m}}|S(\alpha)|^{\frac{4}{3}} d \alpha\right)^{\frac{3}{4}}\left(\int_{\mathfrak{m}}|K(\alpha)|^{4} d \alpha\right)^{\frac{1}{4}}
$$

where the exponents were chosen in such a way, that the first integral will lead to a somewhat natural minor arc problem (in the sense that we have to get a saving slightly better than $B^{-d}$ ) as we will see later. And additionally, the integral in the second term may be easily bounded by

Lemma 2.1.

$$
\int_{\mathfrak{m}}|K(\alpha)|^{4} d \alpha \ll N^{\varepsilon}\left|\mathcal{E}_{N}\right|^{2}
$$


ProOF. For an upper bound we may complete the integral over $\mathfrak{m}$ to a integral over all of $[0,1)$. Using orthogonality, we can interpret the arising integral as the number of solutions to the equation $n_{1}-n_{2}=n_{3}-n_{4}$, where $n_{i} \in \mathcal{E}_{N}$.

This is where the special shape of the set $\mathcal{A}$ of numbers we are trying to represent comes into play. In our case, the $n_{i}$ are values of a quadratic polynomial $\mathfrak{q}(\mathbf{y})$. By completing squares, we see that all we have to do is to bound the number of solutions to $y_{1}^{2}-y_{2}^{2}=y_{3}^{2}-y_{4}^{2}$, where $y_{i}$ are restricted to a suitable set $Y$ of the same cardinality as $\mathcal{E}_{N}$. Both sides of this equation factorize and a usual divisor function estimate yields the desired bound $N^{\varepsilon}\left|\mathcal{E}_{N}\right|^{2}$ as in [5, eq. (3.16)].

Inserting this into (2.7) leads to the bound on the exceptional set of the form

$$
\left|\mathcal{E}_{N}\right| \ll B^{-2 s+2 d+\varepsilon}\left(\int_{\mathfrak{m}}|S(\alpha)|^{\frac{4}{3}} d \alpha\right)^{\frac{3}{2}} .
$$

The next part of the investigation will be to prove the minor arc estimate

$$
\int_{\mathfrak{m}}|S(\alpha)|^{\frac{4}{3}} d \alpha \ll B^{\frac{4}{3} s-d-\delta},
$$

for some $\delta>0$.

Note. This can be seen as a minor arc integral over a polynomial in essentially $\frac{4}{3} s$ variables. So the general idea is, that whenever the circle method is able to handle the minor arc contribution of a special class of polynomials in $s \geq s_{0}$ variables, we can hope to apply the method to that class of polynomials in $s \geq \frac{3}{4} s_{0}$ variables, as will be discussed in chapter 6 .

We conclude this chapter by summing up what we have found so far. Inserting (2.9) into (2.8) yields

$$
\begin{aligned}
\left|\mathcal{E}_{N}\right| & \ll B^{\frac{d}{2}-\frac{3}{2} \delta+\varepsilon} \\
& \ll N^{\frac{1}{2}-\Delta}
\end{aligned}
$$

where $0<\Delta<\frac{3}{2 d} \delta$.

Since $\left|\mathcal{A}_{N}\right| \gg N^{\frac{1}{2}}$, we have

$$
\frac{\left|\mathcal{E}_{N}\right|}{\left|\mathcal{A}_{N}\right|} \ll N^{-\Delta} \rightarrow 0, \text { for } N \rightarrow \infty,
$$

and we can deduce that almost all $n \in \mathcal{A}$ are represented by $f$.

This method will act as the basis for the proofs of our theorems, so we state it as 
Theorem 2.2 (Prototype). Let $f \in \mathbb{Z}\left[x_{1}, \ldots, x_{s}\right]$ be a polynomial of degree $d$, such that

1. for almost all $n \in \mathcal{A}, n \sim N$ we have the major arc estimate

$$
\int_{\mathfrak{M}} S(\alpha) \mathrm{e}(-\alpha n) d \alpha \gg B^{s-d-\varepsilon}
$$

and

2. the minor arc estimate

$$
\int_{\mathfrak{m}}|S(\alpha)|^{\frac{4}{3}} d \alpha \ll B^{\frac{4}{3} s-d-\delta},
$$

for some $\delta>0$. Then almost all $n \in \mathcal{A}$ are represented by $f$. 


\section{Forms of degree $d$}

The following chapter is dedicated to the proof of Theorem 1.1.

The solvability for linear equations is trivial unless there are $p$-adic obstructions. Thus we can assume $f \in \mathbb{Z}\left[x_{1}, \ldots, x_{s}\right]$ to be a form of degree $d \geq 2$ and $\mathcal{A}=\{\mathfrak{q}(y): y \in$ $\mathbb{Z}\} \cap \mathbb{N}$, the positive values of a quadratic polynomial $\mathfrak{q} \in \mathbb{Z}[y]$ with positive leading coefficient. Using the results of the former chapter, we are left with the task to ensure the validity of the major arc estimate (2.11) and the minor arc estimate (2.12) in the Prototype Theorem 2.2.

First of all, we have to choose suitable major arcs. Therefore let $Q=B^{(d-1) \theta}$ for a small parameter $0<\theta<1$ and define the major arcs by

$$
\mathfrak{M}(\theta):=\bigcup_{q \leq Q} \bigcup_{a=1}^{q}{ }^{*}\left[\frac{a}{q}-B^{-d} Q, \frac{a}{q}+B^{-d} Q\right],
$$

and the corresponding minor $\operatorname{arcs}$ as $\mathfrak{m}(\theta):=[0,1) \backslash \mathfrak{M}(\theta)$, which should both be read as being defined modulo 1 .

As prototype for the weight function in (2.1) we choose the indicator function

$$
\omega(t)= \begin{cases}1 & \text { for }-1<t<1 \\ 0 & \text { otherwise }\end{cases}
$$

Let $\mathbf{x}_{0}=0$, such that the weight function $\mathrm{w}_{B}(\mathbf{x})$ is just the indicator function of the box $\mathcal{B}=\left\{\mathbf{x} \in \mathbb{Z}^{s}:\left|x_{i}\right|<\rho, i=1, \ldots, s\right\}$.

We base our work on the results of Birch [1]. Therefore it will be helpful to additionally define the slightly smaller major arcs

$$
\mathfrak{M}^{\prime}(\theta):=\bigcup_{q \leq Q} \bigcup_{a=1}^{q}\left[\frac{a}{q}-\frac{Q}{2 q B^{d}}, \frac{a}{q}+\frac{Q}{2 q B^{d}}\right],
$$

and the corresponding minor arcs as $\mathfrak{m}^{\prime}(\theta):=[0,1) \backslash \mathfrak{M}^{\prime}(\theta)$ modulo 1 .

The main ingredient to our proof will be [1, Lemma 4.3], which we state as

Lemma 3.1. Let $s-\operatorname{dim} \operatorname{sing} f=2^{d-1} K$, then for $\alpha \in \mathfrak{m}^{\prime}(\theta)$ we have

$$
|S(\alpha)| \ll B^{s-K \theta+\varepsilon} \text {. }
$$

Note that in our case we have $K>\frac{3}{2}(d-1)$ by the assumptions in Theorem 1.1. 


\subsection{Minor Arcs}

For the treatment of the minor arcs, we use a pruning argument similar to Birch. We begin by fixing $\delta>0$ and $\theta_{0}>0$ such that

$$
1>\delta+6 d \theta_{0} \quad \text { and } \quad \frac{4}{3} K-2(d-1)>2 \delta \theta_{0}^{-1} .
$$

For this choice we have

Lemma 3.2.

$$
\int_{\mathfrak{m}^{\prime}\left(\theta_{0}\right)}|S(\alpha)|^{\frac{4}{3}} d \alpha \ll B^{\frac{4}{3} s-d-\delta} .
$$

Proof. Choose a sequence $\theta_{T}>\cdots>\theta_{0}$ with

$$
d=2(d-1) \theta_{T} \quad \text { and } \quad \frac{1}{2} \delta>2(d-1)\left(\theta_{t+1}-\theta_{t}\right) \quad \text { for } 0 \leq t<T .
$$

Note that this choice does not dependent on $B$ and can be done with $T \ll 1$.

Now Lemma 3.1 together with (3.1) yields

$$
\begin{aligned}
\int_{\mathfrak{m}^{\prime}\left(\theta_{T}\right)}|S(\alpha)|^{\frac{4}{3}} d \alpha & \ll B^{\frac{4}{3}\left(s-K \theta_{T}\right)+\varepsilon} \\
& \ll B^{\frac{4}{3} s-d-\delta} .
\end{aligned}
$$

We successively fill the gap between $\mathfrak{m}^{\prime}\left(\theta_{T}\right)$ and $\mathfrak{m}^{\prime}\left(\theta_{0}\right)$ by portions of major arcs $\mathfrak{M}^{\prime}\left(\theta_{t+1}\right) \backslash \mathfrak{M}^{\prime}\left(\theta_{t}\right) \subset \mathfrak{m}^{\prime}\left(\theta_{t}\right)$ for $0 \leq t<T$.

The size of $\mathfrak{M}^{\prime}\left(\theta_{t+1}\right)$ can be estimated by $\left|\mathfrak{M}^{\prime}\left(\theta_{t+1}\right)\right| \ll B^{-d+2(d-1) \theta_{t+1}}$. So

$$
\begin{aligned}
\int_{\mathfrak{M}^{\prime}\left(\theta_{t+1}\right) \backslash \mathfrak{M}^{\prime}\left(\theta_{t}\right)}|S(\alpha)|^{\frac{4}{3}} d \alpha & \ll\left|\mathfrak{M}^{\prime}\left(\theta_{t+1}\right)\right| B^{\frac{4}{3}\left(s-K \theta_{t}\right)+\varepsilon} \\
& \ll B^{\frac{4}{3} s-d-\frac{4}{3} K \theta_{t}+2(d-1) \theta_{t+1}+\varepsilon} \\
& \ll B^{\frac{4}{3} s-d-\frac{4}{3} K \theta_{t}+2(d-1) \theta_{t}+\frac{1}{2} \delta+\varepsilon} \\
& \ll B^{\frac{4}{3} s-d-\frac{3}{2} \delta} .
\end{aligned}
$$

Note that $\int_{\mathfrak{m}(\theta)}|S(\alpha)|^{\frac{4}{3}} d \alpha \ll \int_{\mathfrak{m}^{\prime}(\theta)}|S(\alpha)|^{\frac{4}{3}} d \alpha$. So we are free to choose the slightly larger major arcs $\mathfrak{M}$ instead of $\mathfrak{M}^{\prime}$ in the following chapter. 


\subsection{Major Arcs}

We will work with the major $\operatorname{arcs} \mathfrak{M}:=\mathfrak{M}\left(\theta_{0}\right)$, where $\theta_{0}$ was defined by (3.1). We define the exponential sum $S(q, a)=\sum_{\mathbf{x}(q)} \mathrm{e}(a f(\mathbf{x}) / q)$.

Following the lines of Birch [1, §5], we immediately get

$$
\int_{\mathfrak{M}} S(\alpha) \mathrm{e}(-\alpha n) d \alpha=\mathfrak{S}(n) \mathfrak{J}\left(B^{-d} n\right) B^{s-d}+\mathrm{O}\left(B^{s-d-\delta}\right),
$$

for some $\delta>0$, as long as we are able to ensure the absolute convergence of the singular series

$$
\mathfrak{S}(n)=\sum_{q=1}^{\infty} q^{-n} \sum_{a=1}^{q}{ }^{*} S(q, a) \mathrm{e}(-a n / q),
$$

and the singular integral

$$
\mathfrak{J}(\nu)=\int_{\mathbb{R}} \mathrm{e}(-\beta \nu) \int_{\mathbb{R}^{s}} \mathrm{e}(\beta f(\mathbf{x})) \mathrm{w}(\mathbf{x}) d \mathbf{x} d \beta .
$$

\subsubsection{Singular Series}

Applying Lemma 3.1 to the special case $S(q, a)=S(a / q)$ with $B=q$, we get

Lemma 3.3. For $(q ; a)=1$ we have

$$
|S(q, a)| \ll q^{s-\frac{K}{d-1}+\varepsilon} \ll q^{s-\frac{3}{2}-\delta},
$$

for some $\delta>0$, since $K>\frac{3}{2}(d-1)$.

Define $A_{n}(q)=q^{-s} \sum_{a=1}^{q}{ }^{*} S(q, a) \mathrm{e}(-a n / q)$, then for the absolute convergence of the singular series (3.3), we have to show $\sum_{q>Q}\left|A_{n}(q)\right| \rightarrow 0$, as $Q \rightarrow \infty$.

First of all we note that $A_{n}(q)$ is a multiplicative function in $q$ by [13, Lemma 5.1].

Now for $t \in \mathbb{Z}$ coprime to $q$, we have $f(t \mathbf{x})=t^{d} f(\mathbf{x})$ and when $\mathbf{x}$ runs through all residue classes modulo $q$, the same holds true for $t \mathbf{x}$. Thus

$$
S(q, a)=\sum_{\mathbf{x}(q)} \mathrm{e}(a f(\mathbf{x}) / q)=\sum_{t \mathbf{x}(q)} \mathrm{e}(a f(t \mathbf{x}) / q)=\sum_{\mathbf{x}(q)} \mathrm{e}\left(a t^{d} f(\mathbf{x}) / q\right)=S\left(q, a t^{d}\right) .
$$


Let $l$ the inverse to $t$ modulo $q$, so $l t \equiv 1(q)$, then

$$
A_{n l^{d}}(q)=q^{-s} \sum_{a=1}^{q} S(q, a) \mathrm{e}\left(-a l^{d} n / q\right)
$$

and since $a t^{d}$ again runs through all residue classes modulo $q$, we have

$$
A_{n l^{d}}(q)=q^{-s} \sum_{a t^{d}=1}^{q}{ }^{*} S\left(q, a t^{d}\right) \mathrm{e}\left(-a t^{d} l^{d} n / q\right)=q^{-s} \sum_{a=1}^{q}{ }^{*} S(q, a) \mathrm{e}(-a n / q)=A_{n}(q) .
$$

So we have

$$
\varphi(q) A_{n}(q)=\sum_{l=1}^{q} A_{n l^{d}}(q)=q^{-s} \sum_{a=1}^{q} S(q, a) \sum_{l=1}^{q} \mathrm{e}\left(-a n l^{d} / q\right),
$$

where $\varphi(q)$ is Euler's phi function.

The innermost sum can be estimated using the following result from Hua [20].

Lemma 3.4. Let $U(q, b)=\sum_{l=1}^{q} * \mathrm{e}\left(-b l^{d} / q\right)$, then

$$
U(q, b) \ll q^{\frac{1}{2}+\varepsilon}(q ; b)^{\frac{1}{2}}
$$

and

$$
U\left(p^{k}, b\right)=0 \text { for } k>k_{0}(p, b):= \begin{cases}\nu+1 & \text { for } p \nmid d \\ \nu+2 & \text { for } p \mid d\end{cases}
$$

where $p^{\nu} \| b$.

This, together with Lemma 3.3 leads to

$$
\left|A_{n}(q)\right| \ll q^{-1-\delta}(q ; n)^{\frac{1}{2}} .
$$

The factor $(q ; n)^{\frac{1}{2}}$ does no harm, when summed over $q$, since

$$
\sum_{q \sim R}(q ; n)^{\frac{1}{2}}=\sum_{d \mid n} d^{\frac{1}{2}} \sum_{\substack{q \sim R \\ d \mid q}} 1 \leq R \sum_{d \mid n} d^{-\frac{1}{2}} \ll R n^{\varepsilon} .
$$

So we can conclude that the singular series in (3.3) is absolute convergent and can be written as an Euler product

$$
\mathfrak{S}(n)=\prod_{p} \sum_{k=0}^{\infty} A_{n}\left(p^{k}\right) .
$$


Note that by the assumption that there are no $p$-adic obstructions for the $n$ under consideration, we immediately get $\mathfrak{S}(n)>0$. But this is not enough, since for our purpose we need a lower bound uniformly in (almost all) $n$, which we state as

Lemma 3.5. For almost all $n \leq N$, for which there are no p-adic obstructions, we have

$$
\mathfrak{S}(n) \gg N^{-\varepsilon} \text {. }
$$

PROOF. Since $\mathfrak{S}(n)=\prod_{p} \sum_{k=0}^{\infty} A_{n}\left(p^{k}\right)$ is absolute convergent, there exists $c>0$, such that

$$
\prod_{p \geq c} \sum_{k=0}^{\infty} A_{n}\left(p^{k}\right) \gg 1
$$

uniformly in $n \sim N$. For the remaining $p<c$ we can use Lemma 3.4 to find that $A_{n}\left(p^{k}\right)=0$ for $k>k_{0}(p, n)$. Let $M\left(p^{k}, n\right)=\left\{\mathbf{x}\left(\bmod p^{k}\right): f(\mathbf{x}) \equiv n\left(p^{k}\right)\right\}$, then $M\left(p^{k_{0}(p, n)}, n\right)$ is non-empty since there are no $p$-adic obstructions for $n$. We can interpret the factors of the singular series as local densities of solutions and get

$$
\sum_{k=0}^{\infty} A_{n}\left(p^{k}\right)=\sum_{k=0}^{k_{0}(p, n)} A_{n}\left(p^{k}\right)=p^{-(s-1) k_{0}(p, n)}\left|M\left(p^{k_{0}(p, n)}, n\right)\right|>p^{-(s-1) k_{0}(p, n)} .
$$

So $\mathfrak{S}(n) \gg b(n)^{-(s-1)}$, where $b(n)=\prod_{\substack{p^{\nu} \| n \\ p<c}} p^{\nu}$.

All we have to do now, is to show that $b(n)$ is small for almost all $n$. Therefore let $\mathfrak{B}=\{b \leq N: p \mid b \Rightarrow p<c\}$, the set of all $b \leq N$ with prime factors $p<c$. Then the number of $n \leq N$ with $b(n)>X$ is

$$
\begin{aligned}
|\{n \leq N: b(n)>X\}| & =\sum_{\substack{b \in \mathfrak{B} \\
b>X}}|\{n \leq N: b(n)=b\}| \\
& \leq \sum_{\substack{b \in \mathfrak{B} \\
b>X}}|\{n \leq N: b \mid n\}| \\
& \leq \frac{N}{X}|\mathfrak{B}| \ll \frac{N}{X}(\log N)^{c} .
\end{aligned}
$$

On choosing $X=N^{\varepsilon}$, we see that for almost all $n \leq N$ we have $b(n) \ll N^{\varepsilon}$ and therefore for almost all $n$ under consideration $\mathfrak{S}(n) \gg N^{-\varepsilon}$. 
Note. The statement of Lemma 3.5 is non-trivial. If the congruence condition is satisfied for some $n$, then it is for a positive proportion of $n \in \mathcal{A}$. As one can see from the argument above, it is only necessary to check the congruence condition mod $N_{0}:=\prod_{p<c} p^{k_{0}(p)}$. If there is one $n=\mathfrak{q}(y) \equiv f(\mathbf{x}) \bmod N_{0}$, then all $\mathfrak{q}\left(y+k N_{0}\right)$ satisfy the congruence condition.

In fact, only some arithmetic progressions are forbidden by p-adic obstructions. The set of remaining $n \in \mathcal{A}$ still has the same order of magnitude as $\mathcal{A}$.

\subsubsection{Singular Integral}

To ensure the absolute convergence of the singular integral, we write (3.4) as

$$
\mathfrak{J}(\nu)=\int_{\mathbb{R}} \mathrm{e}(-\beta \nu) I_{\mathcal{B}}(\beta) d \beta,
$$

where $I_{\mathcal{B}}(\beta)=\int_{\mathcal{B}} \mathrm{e}(\beta f(\mathbf{x})) d \mathbf{x}$ and $\mathcal{B}=\left\{\mathbf{x} \in \mathbb{Z}^{s}:\left|x_{i}\right|<\rho, i=1, \ldots, s\right\}$ is the box indicated by the weight $\mathrm{w}(\mathbf{x})$.

We want to use [1, Lemma 5.2], which we state as

Lemma 3.6. Let $\mathcal{C}$ be a box contained in $\left\{\mathbf{x} \in \mathbb{Z}^{s}:-1<x_{i}<1, i=1, \ldots, s\right\}$ of side-length $\sigma<1$, then

$$
\left|I_{\mathcal{C}}(\beta)\right| \ll \sigma^{n} \min \left(1,\left(\sigma^{d}|\beta|\right)^{-\frac{K}{d-1}+\varepsilon}\right) .
$$

If we apply this for $1<\phi_{1}<\phi_{2}$, we get

$$
\begin{aligned}
\int_{\phi_{1}}^{\phi_{2}}\left|\mathrm{e}(-\beta \nu) I_{\mathcal{B}}(\beta)\right| d \beta & \ll \int_{\phi_{1}}^{\phi_{2}}|\beta|^{-\frac{K}{d-1}+\varepsilon} d \beta \\
& \ll \int_{\phi_{1}}^{\phi_{2}}|\beta|^{-\frac{3}{2}} d \beta \\
& \ll \phi_{1}^{-\frac{1}{2}},
\end{aligned}
$$

since $K>\frac{3}{2}(d-1)$. Therefore $\mathfrak{J}(\nu)$ is absolutely convergent (uniformly in $\nu$ ). This also implies that $\mathfrak{J}(\nu)$ is continuous, as uniform limit of a sequence of continuous functions.

The final step will be to show that

$$
\mathfrak{J}(1) \gg 1
$$


Then we can ensure that $\mathfrak{J}\left(B^{-d} n\right) \gg 1$ uniformly in $n \in \tilde{\mathcal{E}}_{N}=[N, \tau N] \cap \mathcal{E}$ by choosing $\tau>1$ small enough, since $\mathfrak{J}$ is continuous.

In fact it is a rather standard argument to get (3.8) by Fourier inversion. We refer the reader to $[1, \S 6]$ for more details. Note that the $\operatorname{argument}$ requires $s-\operatorname{dim} \operatorname{sing} f>$ $d+1$, which is the case, since $s-\operatorname{dim} \operatorname{sing} f>\frac{3}{4} 2^{d}(d-1) \geq 3(d-1) \geq d+1$, for $d \geq 2$.

\subsection{Conclusion}

Inserting Lemma 3.5 into (3.2) we get the major arc estimate (2.11) for almost all $n \in \mathcal{A}$ as

$$
\int_{\mathfrak{M}} S(\alpha) \mathrm{e}(-\alpha n) d \alpha \gg B^{s-d-\varepsilon} .
$$

The minor arc estimate (2.12) is obtained via Lemma 3.2. So we can apply our Prototype Theorem 2.2 to deduce Theorem 1.1. 


\section{Cubic Forms}

In this chapter we want to investigate the representation of $n \in \mathcal{A}=\{\mathfrak{q}(y): y \in \mathbb{Z}\} \cap \mathbb{N}$ by non-singular cubic forms $f \in \mathbb{Z}\left[x_{1}, \ldots, x_{s}\right]$ and improve the result of Theorem 1.1 in the case $d=3$ from requiring $s>12$ to $s \geq 8$.

In the setting of chapter 2 we start by defining our major and minor arcs as follows.

For a small parameter $0<\theta<\frac{1}{6}$, we set $Q=B^{\theta}$ and define the major arcs by

$$
\mathfrak{M}:=\bigcup_{q \leq Q} \bigcup_{a=1}^{q}\left[\frac{a}{q}-B^{-3} Q, \frac{a}{q}+B^{-3} Q\right],
$$

and the corresponding minor arcs as $\mathfrak{m}:=[0,1) \backslash \mathfrak{M}$ modulo 1 .

As prototype for the weight function in (2.1) we choose the smooth function

$$
\omega(t)= \begin{cases}\exp \left(\frac{-2}{1-t^{2}}\right) & \text { for }|t|<1, \\ 0 & \text { otherwise }\end{cases}
$$

as in [17] and define the weight function as

$$
\mathrm{w}(\mathbf{x}):=\omega\left(\rho^{-1}\left|\mathbf{x}-\mathbf{x}_{0}\right|\right) \text { and } \mathrm{w}_{B}(\mathbf{x}):=\mathrm{w}\left(\mathbf{x} B^{-1}\right),
$$

where $\rho$ is a fixed, small parameter to control the size of the range in which we search for solutions.

We center the weight function around a point $\mathbf{x}_{0} \in \mathbb{R}^{s}$, which is a solution to $f(\mathbf{x})=1$ with non-vanishing determinant of the Hessian matrix

$$
H(\mathbf{x})=\operatorname{det}\left(\frac{\partial^{2} f}{\partial x_{i} \partial x_{j}}\right)_{1 \leq i, j \leq s} .
$$

Such a point is easy to construct as follows. Let $\mathbf{y} \in \mathbb{R}^{s}$ be a arbitrary point with $f(\mathbf{y})>0$ and $H(\mathbf{y}) \neq 0$. Choose $\mathbf{x}_{0}=f(\mathbf{y})^{-\frac{1}{3}} \mathbf{y}$, then $H\left(\mathbf{x}_{0}\right)=f(\mathbf{y})^{-\frac{1}{3} s} H(\mathbf{y}) \neq 0$ and $f\left(\mathbf{x}_{0}\right)=\left(f(\mathbf{y})^{-\frac{1}{3}}\right)^{3} f(\mathbf{y})=1$. 


\subsection{Preparations}

We write $\alpha=a / q+\beta$ and rewrite the exponential sum (2.2) by changing the order of summation $\mathbf{x} \rightarrow \mathbf{x}+q \mathbf{k}$ and sorting by residue classes modulo $q$ as

$$
S(a / q+\beta)=\sum_{\mathbf{x}(q)} \sum_{\mathbf{k} \in \mathbb{Z}^{s}} \mathrm{e}((a / q+\beta) f(\mathbf{x}+q \mathbf{k})) \mathrm{w}_{B}(\mathbf{x}+q \mathbf{k}) .
$$

Next we apply the Poisson summation formula to get

$$
S(a / q+\beta)=\sum_{\mathbf{x}(q)} \mathrm{e}(a f(\mathbf{x}) / q) \sum_{\mathbf{m} \in \mathbb{Z}^{s}} \int_{\mathbf{y} \in \mathbb{R}^{s}} \mathrm{e}(\beta f(\mathbf{x}+q \mathbf{y})) \mathrm{e}(\mathbf{m} \cdot \mathbf{y}) \mathrm{w}_{B}(\mathbf{x}+q \mathbf{y}) d \mathbf{y} .
$$

On substituting $\mathbf{t}=\mathbf{x}+q \mathbf{y}$ we can write

$$
S(a / q+\beta)=q^{-s} \sum_{\mathbf{m} \in \mathbb{Z}^{s}} S(q, a, \mathbf{m}) I(\mathbf{m} / q, \beta),
$$

where

$$
S(q, a, \mathbf{m})=\sum_{\mathbf{x}(q)} \mathrm{e}((a f(\mathbf{x})-\mathbf{x} \cdot \mathbf{m}) / q)
$$

and

$$
I(\mathbf{m} / q, \beta)=\int_{\mathbf{t} \in \mathbb{R}^{s}} \mathrm{e}(\beta f(\mathbf{t})) \mathrm{e}(\mathbf{m} \cdot \mathbf{t} / q) \mathrm{w}_{B}(\mathbf{t}) d \mathbf{t}
$$

As large values of $\mathbf{m}$ lead to cancellation in the integral above, we can cut the summation over $\mathbf{m}$ at a suitable parameter $M$ and get [3, Lemma 1]

Lemma 4.1. For $\alpha=\frac{a}{q}+\beta$ we have

$$
S(\alpha)=q^{-s} \sum_{|\mathbf{m}| \leq M} S(q, a, \mathbf{m}) I(\mathbf{m} / q, \beta)+\mathrm{O}(1),
$$

where

$$
M \ll q\left(|\beta| B^{2}+B^{-1}\right)(\log B)^{7} .
$$

Moreover we get the following bound, similar to [17, Lemma 7]. 


\section{Lemma 4.2.}

$$
I(\mathbf{m} / q, \beta) \ll \min \left\{B^{s}, B^{-\frac{s}{2}+\varepsilon}|\beta|^{-\frac{s}{2}}\right\}
$$

It will be useful to collect all necessary estimates for the sum $S(q, a, \mathbf{m})$.

Lemma 4.3. $S(q, a, \mathbf{m})$ is multiplicative. Namely for $(p ; q)=1, a=a_{1} q+a_{2} p$ we have

$$
S(p q, a, \mathbf{m})=S\left(p, q^{3} a_{1}, \mathbf{m}\right) S\left(q, p^{3} a_{2}, \mathbf{m}\right) .
$$

Thus it suffices to estimate $S(q, a, \mathbf{m})$ on prime powers $q=p^{k}$ and combining the estimates via Lemma 4.3.

Consider the projective variety defined by $f(\mathbf{x})=0$. Then the dual variety is a hypersurface, defined by an equation $f^{*}(\mathbf{x})=0$. When estimating $S(p, a, \mathbf{m})$, we get a better bound, if $f^{*}(\mathbf{m})$ does not vanish modulo $p$. Namely we have [15, Lemma 13] or [3, Lemma 5], which we state as

Lemma 4.4. Let $q_{1}$ be square-free and $(a ; q)=1$, then we have

$$
\left|\sum_{a=1}^{q}{ }^{*} S\left(q_{1}, a, \mathbf{m}\right)\right| \leq A^{\omega\left(q_{1}\right)} q_{1}^{\frac{1}{2}+\frac{1}{2} s}\left(q_{1} ; f^{*}(\mathbf{m})\right)^{\frac{1}{2}},
$$

This can be extended to the case of squares as in [17, Lemma 11].

Lemma 4.5. Let $q_{2}$ be the square of a square-free number and $(a ; q)=1$, then we have

$$
\left|\sum_{a=1}^{q}{ }^{*} S\left(q_{2}, a, \mathbf{m}\right)\right| \ll q_{2}^{\frac{1}{2}+\frac{1}{2} s}\left(q_{2} ; f^{*}(\mathbf{m})^{2}\right)^{\frac{1}{2}} .
$$

In the square-full case we have [3, Lemma 7] or or [15, Lemma 14], stated as

Lemma 4.6. Let $q_{3}$ be square-full, then we have

$$
\sum_{0<|\mathbf{m}| \leq M}\left|\sum_{a=1}^{q_{3}} S\left(q_{3}, a, \mathbf{m}\right)\right| \ll q_{3}^{1+\frac{1}{2} s+\varepsilon}\left(q_{3}^{\frac{1}{3} s}+M^{s}\right) .
$$

In the case $f^{*}(\mathbf{m})=0$ we additionally get [3, Lemma 7] or [15, Lemma 16] as 
Lemma 4.7. Let $q_{3}$ be square-full, then we have

$$
\sum_{\substack{0<|\mathbf{m}| \leq M \\ f^{*}(\mathbf{m})=0}}\left|\sum_{a=1}^{q_{3}}{ }^{*} S\left(q_{3}, a, \mathbf{m}\right)\right| \ll q_{3}^{\frac{1}{2}+s+\varepsilon}+M^{s-\frac{3}{2}} q_{3}^{\frac{4}{3}+\frac{1}{2} s+\varepsilon} .
$$

For primes occurring to higher powers $p^{k}$ and $\mathbf{m}=0$, we provide a way to reduce the exponent $k$, using

Lemma 4.8. Let $p$ be a prime and $p^{\gamma} \| 6 D$, where $D$ is the discriminant of $f$. Then for $k>2 \gamma+1$ and $(a ; p)=1$ we have the recursive reduction formula

$$
S\left(p^{k}, a, \mathbf{0}\right)=p^{2 s} S\left(p^{k-3}, a, \mathbf{0}\right) .
$$

Proof. We split the sum over $\mathbf{x}\left(\bmod p^{k}\right)$ into $\mathbf{x}=\mathbf{y}+p^{k-\gamma-1} \mathbf{z}$, where we take $\mathbf{y}\left(\bmod p^{k-\gamma-1}\right)$ and $\mathbf{z}\left(\bmod p^{\gamma+1}\right)$. Using Taylor expansion for $k>2 \gamma+1$ we get

$$
f(\mathbf{x}) \equiv f(\mathbf{y})+p^{k-\gamma-1} \nabla f(\mathbf{y}) \cdot \mathbf{z}\left(\bmod p^{k}\right)
$$

and therefore

$$
\begin{aligned}
S\left(p^{k}, a, \mathbf{0}\right) & =\sum_{\mathbf{x}\left(p^{k}\right)} \mathrm{e}\left(a f(\mathbf{x}) / p^{k}\right) \\
& =\sum_{\mathbf{y}\left(p^{k-\gamma-1}\right)} \mathrm{e}\left(a f(\mathbf{y}) / p^{k}\right) \underbrace{\sum_{\mathbf{z}\left(p^{\gamma+1}\right)} \mathrm{e}\left(a \nabla f(\mathbf{y}) \cdot \mathbf{z} / p^{\gamma+1}\right)}_{=0 \text { for } \nabla f(\mathbf{y}) \neq \mathbf{0}\left(p^{\gamma+1}\right)} .
\end{aligned}
$$

So only terms with $\nabla f(\mathbf{x}) \equiv \mathbf{0}\left(\bmod p^{\gamma+1}\right)$ contribute to the sum.

Due to the non-singularity of $f$ and $p^{\gamma+1} \nmid 6 D$, there are no primitive solutions to $\nabla f(\mathbf{x}) \equiv \mathbf{0}\left(\bmod p^{\gamma+1}\right)$ with $(\mathbf{x} ; p)=1$. So only terms with $\mathbf{x} \equiv \mathbf{0}(\bmod p)$ are left and it is enough to sum over $\mathbf{x}$ of the form $\mathbf{x}=p \mathbf{y}$ with $\mathbf{y}\left(\bmod p^{k-1}\right)$. Furthermore we have $f(p \mathbf{y})=p^{3} f(\mathbf{y})$ and get

$$
S\left(p^{k}, a, \mathbf{0}\right)=\sum_{\mathbf{y}\left(p^{k-1}\right)} \mathrm{e}\left(a f(p \mathbf{y}) / p^{k}\right)=\sum_{\mathbf{y}\left(p^{k-1}\right)} \mathrm{e}\left(a f(\mathbf{y}) / p^{k-3}\right) .
$$

Again splitting $\mathbf{y}=\mathbf{y}_{\mathbf{1}}+p^{k-3} \mathbf{y}_{\mathbf{2}}$ and using $f\left(\mathbf{y}_{\mathbf{1}}+p^{k-3} \mathbf{y}_{\mathbf{2}}\right) \equiv f\left(\mathbf{y}_{\mathbf{1}}\right)\left(\bmod p^{k-3}\right)$ yields the desired result

$$
\begin{aligned}
S\left(p^{k}, a, \mathbf{0}\right) & =\sum_{\mathbf{y}_{\mathbf{1}}\left(p^{k-3}\right)} \sum_{\mathbf{y}_{\mathbf{2}}\left(p^{2}\right)} \mathrm{e}\left(a f\left(\mathbf{y}_{\mathbf{1}}+p^{k-3} \mathbf{y}_{\mathbf{2}}\right) / p^{k-3}\right) \\
& =\sum_{\mathbf{y}_{\mathbf{1}}\left(p^{k-3}\right)} \mathrm{e}\left(a f\left(\mathbf{y}_{\mathbf{1}}\right) / p^{k-3}\right) \sum_{\mathbf{y}_{\mathbf{2}}\left(p^{2}\right)} 1 \\
& =p^{2 s} S\left(p^{k-3}, a, \mathbf{0}\right) .
\end{aligned}
$$


Using this we can formulate the general bound as follows.

Lemma 4.9. For $(a ; q)=1$ we have

$$
|S(q, a, \mathbf{0})| \ll q^{\frac{2}{3} s+\varepsilon} .
$$

Proof. For $q=1$ the Lemma is trivial. The case $q=p$ prime is handled by Deligne [14, Theorem 8.4], as well as Lemma 4.4. The case $q=p^{2}$ follows directly from Lemma 4.5 . For $q=p^{k}$ with $k>2$ we may apply Lemma 4.8 for all $p \nmid 6 D$ to recursively reduce to one of the cases above. For the remaining finitely many $p$, we can apply Lemma 4.8 as long as $k>2 \gamma+1$ and for the remaining finitely many $k \leq 2 \gamma+1$ the Lemma holds trivially. For $q$ consisting of different prime powers, we can use Lemma 4.3 to reduce to the cases above.

\subsection{Major Arcs}

Note that for $\alpha \in \mathfrak{M}$ the summation over $\mathbf{m}$ in Lemma 4.1 is restricted to only one summand, namely $\mathbf{m}=\mathbf{0}$, so the integral over the major arcs (2.11) can be written as

$$
\int_{\mathfrak{M}} S(\alpha) \mathrm{e}(-\alpha n) d \alpha=\sum_{q \leq Q} q^{-s} \sum_{a=1}^{q} S(q, a, \mathbf{0}) \mathrm{e}(-a n / q) \int_{|\beta| \leq Q B^{-3}} I(\mathbf{0}, \beta) \mathrm{e}(-\beta n) d \beta+\mathrm{O}(1) .
$$

We can estimate the error from completing the integral using Lemma 4.2 as

$$
\ll \int_{Q B^{-3}}^{\infty}|I(\mathbf{0}, \beta)| d \beta \ll B^{-\frac{s}{2}+\varepsilon} \int_{Q B^{-3}}^{\infty}|\beta|^{-\frac{s}{2}} d \beta \ll B^{s-3+\varepsilon} Q^{-\frac{s}{2}+1}
$$

which produces a total error

$$
\sum_{q \leq Q} q^{-s} \sum_{a=1}^{q}|S(q, a, \mathbf{0})| \int_{Q B^{-3}}^{\infty}|I(\mathbf{0}, \beta)| d \beta \ll B^{s-3+\varepsilon} Q^{-\frac{s}{2}+3}
$$

where we have used the trivial bound $|S(q, a, \mathbf{0})| \leq q^{s}$. This is satisfactory for $s \geq 7$. 
Now it is again a rather standard substitution (as in $[13, \S 16]$ similar to section 3.2 .2 ) to rewrite the integral in the form $\mathfrak{J}\left(B^{-3} n\right) B^{s-3}$, where

$$
\mathfrak{J}(\nu)=\int_{\mathbb{R}} \mathrm{e}(-\beta \nu) \int_{\mathbb{R}^{s}} \mathrm{e}(\beta f(\mathbf{x})) \mathrm{w}(\mathbf{x}) d \mathbf{x} d \beta
$$

is the singular integral. Which is a continuous function in $\nu$ and $\mathfrak{J}(1)$ can be interpreted as suitable volume of the set $\left\{f(\mathbf{x})=1, \mathbf{x} \in \mathbb{R}^{s}\right\}$, which is non-zero, since $f$ is homogeneous of odd degree and therefore has no real obstructions. So for suitably small $\tau>1$, we can ensure that $\mathfrak{J}\left(B^{-3} n\right) \gg 1$ uniformly in $n \in \tilde{\mathcal{E}}_{N}=[N, \tau N] \cap \mathcal{E}$.

Next we want to complete the sum over $q$ in (4.3). Therefore we write

$$
A_{n}(q)=q^{-s} \sum_{a=1}^{q} S(q, a, 0) \mathrm{e}(-a n / q) .
$$

As in section 3.2.1 we get

$$
\begin{aligned}
A_{n}(q) & =\varphi(q)^{-1} q^{-s} \sum_{a=1}^{q} S(q, a, \mathbf{0}) U(q, a n) \\
& \ll q^{-\frac{s}{3}+\frac{1}{2}+\varepsilon}(q ; n),
\end{aligned}
$$

using Lemma 4.9.

So the error on completing the sum is

$$
\sum_{q>Q} q^{-s}\left|\sum_{a=1}^{q} S(q, a, \mathbf{0}) \mathrm{e}(-a n / q)\right| \ll \sum_{q>Q} q^{-\frac{s}{3}+\frac{1}{2}+\varepsilon}(q ; n) \ll Q^{-\frac{s}{3}+\frac{3}{2}+\varepsilon} n^{\varepsilon},
$$

which is satisfactory for $s \geq 5$. With exactly the same arguments that lead to Lemma 3.5 in section 3.2.1, we find that the singular series

$$
\mathfrak{S}(n)=\sum_{q} q^{-s} \sum_{a=1}^{*} S(q, a, \mathbf{0}) \mathrm{e}(-a n / q)
$$

is $\gg n^{-\varepsilon}$ for almost all $n \leq N$, as long as there are no $p$-adic obstructions.

We sum up what we have found as the main result for this chapter.

Lemma 4.10. For $s \geq 7$ we have

$$
\int_{\mathfrak{M}} S(\alpha) \mathrm{e}(-\alpha n)=\mathfrak{S}(n) \mathfrak{J}\left(B^{-3} n\right) B^{s-3}+\mathrm{O}\left(B^{s-3-\frac{\theta}{2}+\varepsilon}\right),
$$

where the singular series $\mathfrak{S}(n) \gg n^{\varepsilon}$ and the singular integral $\mathfrak{J}\left(B^{-3} n\right) \gg 1$ for almost all $n \in \mathcal{E}_{N}$ without p-adic obstructions. 


\subsection{Minor Arcs}

To treat the minor arc integral (2.12), we apply Dirichlet's approximation theorem and write $\alpha \in \mathfrak{m}$ as $\alpha=a / q+\beta$, where $(a ; q)=1, q \leq \hat{Q}$ and $|\beta| \leq(q \hat{Q})^{-1}$, which will be optimal for the parameter $\hat{Q}=B^{\frac{3}{2}}$.

Note that $\alpha \in \mathfrak{m}$ implies $q>Q$ or $|\beta|>Q B^{-3}$, which will be indicated by $\star$ in the following integration over $\beta$.

We get

$$
\int_{\mathfrak{m}}|S(\alpha)|^{\frac{4}{3}} d \alpha \ll \sum_{q \leq \hat{Q}} \sum_{a=1}^{q} \int_{\beta}^{\star}|S(a / q+\beta)|^{\frac{4}{3}} d \beta .
$$

It will be convenient to split the summation over $q$ into $\mathrm{O}(\log B)$ intervals of the form $R<q \leq 2 R$. So we can bound the minor arc integral by

$$
\ll B^{\varepsilon} \max _{R \leq \hat{Q}} \sum_{q \sim R} \sum_{a=1}^{q} \int_{\beta}^{\star}|S(a / q+\beta)|^{\frac{4}{3}} d \beta .
$$

Now follows a discussion of the various ranges for $R$ and $\beta$.

We begin by considering the case $|\beta| \leq B^{-3}$. Note that being on the minor arcs, we have $R>Q$.

Applying Hölder's inequality to (4.4), we get

$$
\ll B^{-1+\varepsilon} \max _{Q<R \leq \hat{Q}} R^{\frac{2}{3}}\left(\sum_{q \sim R} \sum_{a=1}^{q} * \int_{|\beta| \leq B^{-3}}|S(a / q+\beta)|^{2} d \beta\right)^{\frac{2}{3}} .
$$

Expanding the squares, we find that $|S(\alpha)|^{2}=S_{2}(\alpha)$, where $S_{2}$ is the exponential sum corresponding to the polynomial $g(\mathbf{x}, \mathbf{y})=f(\mathbf{x})-f(\mathbf{y}) \in \mathbb{Z}\left[x_{1}, \ldots, x_{s}, y_{1}, \ldots, y_{s}\right]$ in $2 s$ variables. Note that $g$ is non-singular again, therefore we may use all our previous results with $s$ replaced by $2 s$ for $g$ instead of $f$.

Applying Lemma 4.1 to $S_{2}(\alpha)$, we get

$$
S_{2}(a / q+\beta)=q^{-2 s} \sum_{|\mathbf{m}| \leq M} S_{2}(q, a, \mathbf{m}) I_{2}(\mathbf{m} / q, \beta)+\mathrm{O}(1)
$$


where $S_{2}(q, a, \mathbf{m})$ and $I_{2}(\nu, \beta)$ are defined to correspond to $g$ instead of $f$ accordingly.

The contribution coming from the error term $\mathrm{O}(1)$ to $(4.5)$ is negligible and we find that these ranges of the minor arcs can be estimated by

$$
\begin{aligned}
& \ll B^{-1+\varepsilon} \max _{Q<R \leq \hat{Q}} R^{\frac{2}{3}}\left(\sum_{q \sim R} q^{-2 s} \sum_{|\mathbf{m}| \leq M} \sum_{a=1}^{q} S_{2}(q, a, \mathbf{m}) \int_{|\beta| \leq B^{-3}} I_{2}(\mathbf{m} / q, \beta) d \beta\right)^{\frac{2}{3}} \\
& \ll B^{\frac{4}{3} s-3+\varepsilon} \max _{Q<R \leq \hat{Q}} R^{\frac{2}{3}}\left(\sum_{q \sim R} q^{-2 s} \sum_{0<|\mathbf{m}| \leq M}\left|\sum_{a=1}^{q} S_{2}(q, a, \mathbf{m})\right|\right)^{\frac{2}{3}},
\end{aligned}
$$

using the trivial bound $B^{2 s}$ from Lemma 4.2 for $I_{2}\left(\frac{\mathbf{m}}{q}, \beta\right)$.

The term with $\mathbf{m}=\mathbf{0}$ is reminiscent of the major arc approximation and shall be treated first.

Here $S_{2}(q, a, \mathbf{0})$ can be bounded by Lemma 4.9. The contribution is

$$
\begin{aligned}
& \ll B^{\frac{4}{3} s-3+\varepsilon} \max _{Q<R \leq \hat{Q}} R^{2-\frac{4}{9} s} \\
& \ll B^{\frac{4}{3} s-3+\varepsilon} Q^{\frac{18-4 s}{9}},
\end{aligned}
$$

which is satisfactory for $s \geq 5$.

For the terms with $\mathbf{m} \neq \mathbf{0}$ in (4.7), we have $|\mathbf{m}| \leq M \ll q B^{-1+\varepsilon}$ by (4.2), so we only have to consider the $q \gg B$, which will contribute

$$
\ll B^{\frac{4}{3} s-3+\varepsilon} \max _{B<R \leq \hat{Q}} R^{\frac{2}{3}}\left(\sum_{q \sim R} q^{-2 s} \sum_{0<|\mathbf{m}| \leq M}\left|\sum_{a=1}^{q}{ }^{*} S_{2}(q, a, \mathbf{m})\right|\right)^{\frac{2}{3}} .
$$

We write

$$
q=q_{1} q_{2} q_{3},
$$

where $q_{1}, q_{2}, q_{3}$ are pairwise co-prime, $q_{1}$ is square-free, $q_{2}$ is the square of a squarefree number and $q_{3}$ contains all prime factors that occur at least to the power 3 in $q$. Using the multiplicativity of $S_{2}$ in Lemma 4.3 , we get

$$
\left|\sum_{a=1}^{q}{ }^{*} S_{2}(q, a, \mathbf{m})\right| \ll\left|\sum_{a_{1}=1}^{q_{1}}{ }^{*} S_{2}\left(q_{1}, a_{1}, \mathbf{m}\right)\right|\left|\sum_{a_{2}=1}^{q_{2}} * S_{2}\left(q_{2}, a_{2}, \mathbf{m}\right)\right|\left|\sum_{a_{3}=1}^{q_{3}} * S_{2}\left(q_{3}, a_{3}, \mathbf{m}\right)\right| .
$$


The three summands can be estimated using Lemmata 4.4 to 4.7.

For the moment, we restrict our attention to those $\mathbf{m}$ in $(4.7)$, with $g^{*}(\mathbf{m}) \neq 0$. We employ Lemmata 4.4 and 4.5 together with (4.9) and get the bound

$\ll B^{\frac{4}{3} s-3+\varepsilon} \max _{B<R \leq \hat{Q}} R^{\frac{2}{3}}\left(\sum_{q \sim R}\left(q_{1} q_{2}\right)^{-s+\frac{1}{2}} q_{3}^{-2 s} \sum_{0<|\mathbf{m}| \leq M}\left(q_{1} q_{2} ; g^{*}(\mathbf{m})\right)^{\frac{1}{2}}\left|\sum_{a=1}^{q_{3}} S_{2}\left(q_{3}, a, \mathbf{m}\right)\right|\right)^{\frac{2}{3}}$.

As in (3.6), the gcd only contributes $q^{\varepsilon}$, when summed over $q$. For the innermost sum we use Lemma 4.6 and are left with

$$
\ll B^{\frac{4}{3} s-3+\varepsilon} \max _{B<R \leq \hat{Q}} R^{\frac{2}{3}}\left(\sum_{q \sim R}\left(q_{1} q_{2}\right)^{-s+\frac{1}{2}+\varepsilon} q_{3}^{1-s+\varepsilon}\left(q_{3}^{\frac{2}{3} s}+M^{2 s}\right)\right)^{\frac{2}{3}} .
$$

The terms involving $q_{3}$ contribute

$$
\begin{aligned}
& \ll B^{\frac{4}{3} s-3+\varepsilon} \max _{B<R \leq \hat{Q}} R^{\frac{2}{3}}\left(\sum_{q \sim R}\left(q_{1} q_{2}\right)^{-s+\frac{1}{2}+\varepsilon} q_{3}^{1-\frac{1}{3} s+\varepsilon}\right)^{\frac{2}{3}} \\
& \ll B^{\frac{4}{3} s-3+\varepsilon} \max _{B<R \leq \hat{Q}} R^{\frac{2}{3}}\left(R^{\frac{4}{3}-\frac{1}{3} s+\varepsilon}\right)^{\frac{2}{3}} \\
& \ll B^{\frac{4}{3} s-3+\varepsilon} \max _{B<R \leq \hat{Q}} R^{\frac{14}{9}-\frac{2}{9} s+\varepsilon} \\
& \ll B^{\frac{4}{3} s-3-\frac{2}{9}+\varepsilon}
\end{aligned}
$$

for $s \geq 8$.

The terms involving $M$ contribute

$$
\begin{aligned}
& \ll B^{\frac{4}{3} s-3+\varepsilon} \max _{B<R \leq \hat{Q}} R^{\frac{2}{3}}\left(\sum_{q \sim R}\left(q_{1} q_{2}\right)^{-s+\frac{1}{2}+\varepsilon} q_{3}^{1-s+\varepsilon}\left(q B^{-1+\varepsilon}\right)^{2 s}\right)^{\frac{2}{3}} \\
& \ll B^{-3+\varepsilon} \max _{B<R \leq \hat{Q}} R^{\frac{2}{3}}\left(\sum_{q \sim R}\left(q_{1} q_{2}\right)^{s+\frac{1}{2}+\varepsilon} q_{3}^{1+s+\varepsilon}\right)^{\frac{2}{3}} \\
& \ll B^{-3+\varepsilon} \max _{B<R \leq \hat{Q}} R^{\frac{2}{3} s+\frac{5}{3}+\varepsilon} \\
& \ll B^{-3+\varepsilon} \hat{Q}^{\frac{2}{3} s+\frac{5}{3}+\varepsilon} \ll B^{\frac{4}{3} s-3-\frac{2 s-15}{6}+\varepsilon} \ll B^{\frac{4}{3} s-3-\frac{1}{6}+\varepsilon},
\end{aligned}
$$

for $s \geq 8$. 
For the remaining $\mathbf{m}$ in $(4.7)$ with $g^{*}(\mathbf{m})=0$ we proceed similarly, but instead of only using Lemma 4.6 we can also employ Lemma 4.7 and get the bound

$$
\begin{aligned}
& \ll B^{\frac{4}{3} s-3+\varepsilon} \max _{B<R \leq \hat{Q}} R^{\frac{2}{3}}\left(\sum_{q \sim R}\left(q_{1} q_{2}\right)^{-s+1} q_{3}^{-2 s} \sum_{\substack{0<|\mathbf{m}| \leq M \\
g^{*}(\mathbf{m})=0}}\left|\sum_{a=1}^{q_{3}} S_{2}\left(q_{3}, a, \mathbf{m}\right)\right|\right)^{\frac{2}{3}} \\
& \ll B^{\frac{4}{3} s-3+\varepsilon} \max _{B<R \leq \hat{Q}} R^{\frac{2}{3}}\left(\sum_{q \sim R}\left(q_{1} q_{2}\right)^{-s+1} \min \left(q_{3}^{1-s+\varepsilon}\left(q_{3}^{\frac{2}{3} s}+M^{2 s}\right), q^{\frac{1}{2}+\varepsilon}+q^{\frac{4}{3}-s+\varepsilon} M^{2 s-3}\right)\right)^{\frac{2}{3}} .
\end{aligned}
$$

First we consider the term $q_{3}^{1-\frac{1}{3} s}$ in the minimum. Its contribution is

$$
\begin{aligned}
& \ll B^{\frac{4}{3} s-3+\varepsilon} \max _{B<R \leq \hat{Q}} R^{\frac{2}{3}}\left(\sum_{q \sim R}\left(q_{1} q_{2}\right)^{-s+1} q_{3}^{1-\frac{1}{3} s}\right)^{\frac{2}{3}} \\
& \ll B^{\frac{4}{3} s-3+\varepsilon} \max _{B<R \leq \hat{Q}} R^{\frac{14-2 s}{9}} \\
& \ll B^{\frac{4}{3} s-3+\frac{14-2 s}{9}+\varepsilon} \\
& \ll B^{\frac{4}{3} s-3-\frac{2}{9}+\varepsilon},
\end{aligned}
$$

for $s \geq 8$.

Next we investigate the term $q_{3}^{\frac{4}{3}-s+\varepsilon} M^{2 s-3}$, this contributes

$$
\begin{aligned}
& \ll B^{\frac{4}{3} s-3+\varepsilon} \max _{B<R \leq \hat{Q}} R^{\frac{2}{3}}\left(\sum_{q \sim R}\left(q_{1} q_{2}\right)^{-s+1} q_{3}^{\frac{4}{3}-s+\varepsilon}\left(q B^{-1}\right)^{2 s-3}\right)^{\frac{2}{3}} \\
& \ll B^{-1+\varepsilon} \max _{B<R \leq \hat{Q}} R^{\frac{2}{3}}\left(\sum_{q \sim R}\left(q_{1} q_{2}\right)^{s-2} q_{3}^{-\frac{5}{3}+s+\varepsilon}\right)^{\frac{2}{3}} \\
& \ll B^{-1+\varepsilon} \max _{B<R \leq \hat{Q}} R^{\frac{2}{3} s} \\
& \ll B^{-1+\varepsilon} \hat{Q}^{\frac{2}{3} s} \\
& \ll B^{\frac{4}{3} s-3+\frac{6-s}{3}+\varepsilon},
\end{aligned}
$$

which is satisfactory for $s \geq 7$.

What remains is to consider the terms with

$$
\begin{aligned}
\min \left(q_{3}^{1-s+\varepsilon} M^{2 s}, q_{3}^{\frac{1}{2}+\varepsilon}\right) & \ll \min \left(q_{3}^{1-s+\varepsilon}\left(q B^{-1+\varepsilon}\right)^{2 s}, q_{3}^{\frac{1}{2}+\varepsilon}\right) \\
& \ll\left(q_{3}^{1-s+\varepsilon}\left(q B^{-1+\varepsilon}\right)^{2 s}\right)^{A}\left(q_{3}^{\frac{1}{2}+\varepsilon}\right)^{1-A} .
\end{aligned}
$$


These contribute

$$
\begin{aligned}
& \ll B^{\frac{4}{3} s-3+\varepsilon} \max _{B<R \leq \hat{Q}} R^{\frac{2}{3}}\left(\sum_{q \sim R}\left(q_{1} q_{2}\right)^{-s+1}\left(q_{3}^{1-s+\varepsilon}\left(q B^{-1+\varepsilon}\right)^{2 s}\right)^{A}\left(q_{3}^{\frac{1}{2}+\varepsilon}\right)^{1-A}\right)^{\frac{2}{3}} \\
& \ll B^{\frac{4}{3} s-3-\frac{4}{3} A s+\varepsilon} \max _{B<R \leq \hat{Q}} R^{\frac{2}{3}}\left(\sum_{q \sim R}\left(q_{1} q_{2}\right)^{-s+1+2 A s} q_{3}^{\frac{1}{2} A+\frac{1}{2}+A s+\varepsilon}\right)^{\frac{2}{3}} .
\end{aligned}
$$

It turns out that the optimal choice for $A$ is $A=1-\frac{4}{6 s-3}$, which leads to

$$
\begin{aligned}
& \ll B^{\frac{4}{3} s-3-\frac{4}{3}\left(1-\frac{4}{6 s-3}\right) s+\varepsilon} \max _{B<R \leq \hat{Q}} R^{\frac{2}{3}}\left(R^{s+\frac{2}{3}-\frac{4}{6 s-3}}\right)^{\frac{2}{3}} \\
& \ll B^{\frac{4}{3} s-3-\frac{4}{3}\left(1-\frac{4}{6 s-3}\right) s+\varepsilon} \max _{B<R \leq \hat{Q}} R^{\frac{2}{3} s+\frac{10}{9}-\frac{2}{3 s-1}} \\
& \ll B^{\frac{4}{3} s-3-\frac{4}{3}\left(1-\frac{4}{6 s-3}\right) s+\varepsilon} \hat{Q}^{\frac{2}{3} s+\frac{10}{9}-\frac{2}{3 s-1}} \\
& \ll B^{\frac{4}{3} s-3-\frac{4}{3}\left(1-\frac{4}{6 s-3}\right) s+\varepsilon} B^{s+\frac{5}{3}-\frac{3}{3 s-1}} \\
& \ll B^{\frac{4}{3} s-3-\frac{23-3 s}{9}-\frac{4}{18 s-9}+\varepsilon} \\
& \ll B^{\frac{4}{3} s-3-\frac{1}{9}+\varepsilon}
\end{aligned}
$$

for $s \geq 8$.

We split the remaining range for $\beta$ into $\mathrm{O}(\log B)$ intervals of the form $\phi<|\beta| \leq 2 \phi$. And again apply Hölder's inequality and Lemma 4.1, to get the analog of (4.6) as

$$
\begin{aligned}
& \ll \max _{R, \phi}\left(R^{2} \phi\right)^{\frac{1}{3}}\left(\sum_{q \sim R} q^{-2 s} \sum_{|\mathbf{m}| \leq M} \sum_{a=1}^{q} S_{2}(q, a, \mathbf{m}) \int_{\beta \sim \phi} I_{2}(\mathbf{m} / q, \beta) d \beta\right)^{\frac{2}{3}} \\
& \ll B^{-\frac{2}{3} s+\varepsilon} \max _{R, \phi} R^{\frac{2}{3}} \phi^{-\frac{2}{3} s+1}\left(\sum_{q \sim R} q^{-2 s} \sum_{|\mathbf{m}| \leq M}\left|\sum_{a=1}^{q} S_{2}(q, a, \mathbf{m})\right|\right)^{\frac{2}{3}},
\end{aligned}
$$

where the innermost integral has been estimated by Lemma 4.2 to be $\ll B^{-s+\varepsilon} \phi^{-s+1}$, and the maximum ranges over all $R \leq \hat{Q}$ and $B^{-3}<\phi \leq \frac{1}{R \hat{Q}}$, such that not both $R<Q$ and $\phi<Q B^{-3}$ hold simultaneously.

Next we want to estimate the contribution of the $B^{-3}<\phi \leq Q B^{-3}$ and thus we have $R>Q$. 
For the term with $\mathbf{m}=\mathbf{0}$ we use Lemma 4.9 to bound $S_{2}(q, a, \mathbf{0})$ and get a contribution

$$
\begin{aligned}
& \ll B^{-\frac{2}{3} s+\varepsilon} \max _{R, \phi} R^{-\frac{4}{9} s+2} \phi^{-\frac{2}{3} s+1} \\
& \ll B^{\frac{4}{3} s-3+\varepsilon} Q^{\frac{18-4 s}{9}}
\end{aligned}
$$

which is satisfactory for $s \geq 5$.

For the terms with $\mathbf{m} \neq \mathbf{0}$ in (4.12), we have $|\mathbf{m}| \leq M \ll R \phi B^{2+\varepsilon}$ by (4.2). Again dividing into the $\mathbf{m}$ with $g^{*}(\mathbf{m}) \neq 0$ and those with $g^{*}(\mathbf{m})=0$, the sum over $q$ can be estimated by multiplicativity and the Lemmata from chapter 4.1 as

$$
\begin{aligned}
\sum_{q \sim R} q^{-2 s} \sum_{\substack{|\mathbf{m}| \leq M \\
g^{*}(\mathbf{m}) \neq 0}}\left|\sum_{a=1}^{q} S_{2}(q, a, \mathbf{m})\right| & \ll \sum_{q \sim R}\left(q_{1} q_{2}\right)^{-s+\frac{1}{2}+\varepsilon} q_{3}^{1-s+\varepsilon}\left(q_{3}^{\frac{2}{3} s}+M^{2 s}\right) \\
& \ll \sum_{q \sim R}\left(q_{1} q_{2}\right)^{-s+\frac{1}{2}+\varepsilon} q_{3}^{1-s+\varepsilon}\left(q_{3}^{\frac{2}{3} s}+\left(R \phi B^{2+\varepsilon}\right)^{2 s}\right) \\
& \ll R^{\frac{4}{3}-\frac{1}{3} s}+R^{\frac{3}{2}+s} \phi^{2 s} B^{4 s+\varepsilon}
\end{aligned}
$$

similar to (4.10), where we have written $q=q_{1} q_{2} q_{3}$ as in (4.8).

Inserting (4.15) into (4.12), we see that the contribution from $\mathbf{m}$ with $g^{*}(\mathbf{m}) \neq 0$ is

$$
\begin{aligned}
& \ll B^{-\frac{2}{3} s+\varepsilon} \max _{R, \phi} R^{\frac{2}{3}} \phi^{-\frac{2}{3} s+1}\left(R^{\frac{4}{3}-\frac{1}{3} s}+R^{\frac{3}{2}+s} \phi^{2 s} B^{4 s+\varepsilon}\right)^{\frac{2}{3}} \\
& \ll B^{-\frac{2}{3} s+\varepsilon} \max _{R, \phi}\left(R^{\frac{14-2 s}{9}} \phi^{-\frac{2}{3} s+1}+R^{\frac{5}{3}+\frac{2}{3} s} \phi^{\frac{2}{3} s+1} B^{\frac{8}{3} s+\varepsilon}\right) \\
& \ll B^{\frac{4}{3} s-3+\varepsilon} Q^{\frac{14-2 s}{9}}+B^{-3+\varepsilon} \hat{Q}^{\frac{5}{3}+\frac{2}{3} s} Q^{\frac{2}{3} s+1} \\
& \ll B^{\frac{4}{3} s-3+\varepsilon} Q^{\frac{14-2 s}{9}}+B^{s-\frac{1}{2}+\varepsilon} Q^{\frac{2}{3} s+1} \\
& \ll B^{\frac{4}{3} s-3+\varepsilon} Q^{\frac{14-2 s}{9}}+B^{\frac{4}{3} s-3+\varepsilon+\frac{15-2 s}{6}} Q^{\frac{2}{3} s+1} .
\end{aligned}
$$


For $\mathbf{m}$ with $g^{*}(\mathbf{m})=0$, we get

$$
\begin{aligned}
& \sum_{q \sim R} q^{-2 s} \sum_{\substack{|\mathbf{m}| \leq M \\
g^{*}(\mathbf{m})=0}}\left|\sum_{a=1}^{q} S_{2}(q, a, \mathbf{m})\right| \\
& \ll \sum_{q \sim R}\left(q_{1} q_{2}\right)^{-s+1} \min \left(q_{3}^{1-s+\varepsilon}\left(q_{3}^{\frac{2}{3} s}+M^{2 s}\right), q_{3}^{\frac{1}{2}+\varepsilon}+q_{3}^{\frac{4}{3}-s+\varepsilon} M^{2 s-3}\right) \\
& \ll \sum_{q \sim R}\left(q_{1} q_{2}\right)^{-s+1} q_{3}^{1-\frac{1}{3} s+\varepsilon} \\
& \quad+\sum_{q \sim R}\left(q_{1} q_{2}\right)^{-s+1} q_{3}^{\frac{4}{3}-s+\varepsilon}\left(R \phi B^{2+\varepsilon}\right)^{2 s-3} \\
& \quad+\sum_{q \sim R}\left(q_{1} q_{2}\right)^{-s+1} \min \left(q_{3}^{1-s+\varepsilon}\left(R \phi B^{2+\varepsilon}\right)^{2 s}, q_{3}^{\frac{1}{2}+\varepsilon}\right)
\end{aligned}
$$

as in (4.11).

The contribution of the summand (4.17) to (4.12) is

$$
\begin{aligned}
& \ll B^{-\frac{2}{3} s+\varepsilon} \max _{R, \phi} R^{\frac{2}{3}} \phi^{-\frac{2}{3} s+1}\left(\sum_{q \sim R}\left(q_{1} q_{2}\right)^{-s+1} q_{3}^{1-\frac{1}{3} s+\varepsilon}\right)^{\frac{2}{3}} \\
& \ll B^{-\frac{2}{3} s+\varepsilon} \max _{R, \phi} R^{\frac{2}{3}} \phi^{-\frac{2}{3} s+1}\left(R^{\frac{4-s}{3}}\right)^{\frac{2}{3}} \\
& \ll B^{-\frac{2}{3} s+\varepsilon} \max _{R, \phi} R^{\frac{14-2 s}{9}} \phi^{-\frac{2}{3} s+1} \\
& \ll B^{-\frac{2}{3} s+\varepsilon} \max _{R, \phi} R^{\frac{14-2 s}{9}} \phi^{-\frac{2}{3} s+1} \\
& \ll B^{\frac{4}{3} s-3+\varepsilon} Q^{\frac{14-2 s}{9}} .
\end{aligned}
$$

The contribution of the summand (4.18) to (4.12) is

$$
\begin{aligned}
& \ll B^{-\frac{2}{3} s+\varepsilon} \max _{R, \phi} R^{\frac{2}{3}} \phi^{-\frac{2}{3} s+1}\left(\sum_{q \sim R}\left(q_{1} q_{2}\right)^{-s+1} q_{3}^{\frac{4}{3}-s+\varepsilon}\left(R \phi B^{2+\varepsilon}\right)^{2 s-3}\right)^{\frac{2}{3}} \\
& \ll B^{2 s-4+\varepsilon} \max _{R, \phi} R^{\frac{2}{3} s} \phi^{\frac{2}{3} s-1} \\
& \ll B^{-1+\varepsilon} \hat{Q}^{\frac{2}{3} s} Q^{\frac{2}{3} s-1} \\
& \ll B^{\frac{4}{3} s-3+\frac{6-s}{3}+\varepsilon} Q^{\frac{2}{3} s-1},
\end{aligned}
$$

which is satisfactory for $s \geq 7$ and $\theta$ small enough. 
The minimum in (4.19) is

$$
\begin{aligned}
\min \left(q_{3}^{1-s+\varepsilon} M^{2 s}, q_{3}^{\frac{1}{2}+\varepsilon}\right) & \ll \min \left(q_{3}^{1-s+\varepsilon}\left(R \phi B^{2+\varepsilon}\right)^{2 s}, q_{3}^{\frac{1}{2}+\varepsilon}\right) \\
& \ll\left(q_{3}^{1-s+\varepsilon}\left(R \phi B^{2+\varepsilon}\right)^{2 s}\right)^{A}\left(q_{3}^{\frac{1}{2}+\varepsilon}\right)^{1-A} .
\end{aligned}
$$

So its contribution to (4.12) is

$$
\begin{aligned}
& \ll B^{-\frac{2}{3} s+\varepsilon} \max _{R, \phi} R^{\frac{2}{3}} \phi^{-\frac{2}{3} s+1}\left(\sum_{q \sim R}\left(q_{1} q_{2}\right)^{-s+1}\left(q_{3}^{1-s+\varepsilon}\left(R \phi B^{2+\varepsilon}\right)^{2 s}\right)^{A}\left(q_{3}^{\frac{1}{2}+\varepsilon}\right)^{1-A}\right)^{\frac{2}{3}} \\
& \ll B^{-\frac{2}{3} s+\frac{8}{3} A s+\varepsilon} \max _{R, \phi} R^{\frac{2}{3}} \phi^{-\frac{2}{3} s+\frac{4}{3} A s+1}\left(\sum_{q \sim R}\left(q_{1} q_{2}\right)^{-s+2 A s+1} q_{3}^{\frac{1}{2} A+A s+\frac{1}{2}}\right)^{\frac{2}{3}} .
\end{aligned}
$$

Choosing $A=1-\frac{4}{6 s-3}$ as above, leads to

$$
\begin{aligned}
& \ll B^{2 s-\frac{16}{9}-\frac{16}{18 s-9}+\varepsilon} \max _{R, \phi} R^{\frac{2}{3} s+\frac{10}{9}-\frac{8}{18 s-9}} \phi^{\frac{2}{3} s+\frac{1}{9}-\frac{8}{18 s-9}} \\
& \ll B^{-\frac{19}{9}+\frac{8}{18 s-9}+\varepsilon} \hat{Q}^{\frac{2}{3} s+\frac{10}{9}-\frac{8}{18 s-9}} Q^{\frac{2}{3} s+\frac{1}{9}-\frac{8}{18 s-9}} \\
& \ll B^{\frac{4}{3} s-3+\frac{23-3 s}{9}+\frac{4}{18 s-9}+\varepsilon} Q^{\frac{2}{3} s+\frac{1}{9}-\frac{8}{18 s-9}},
\end{aligned}
$$

which is satisfactory for $s \geq 8$.

We finish our estimates by considering the remaining range $Q B^{-3}<\phi \leq \frac{1}{R \hat{Q}}$ in (4.12).

As in (4.13) the term with $\mathbf{m}=\mathbf{0}$ contributes

$$
\begin{aligned}
& \ll B^{-\frac{2}{3} s+\varepsilon} \max _{R, \phi} R^{-\frac{4}{9} s+2} \phi^{-\frac{2}{3} s+1} \\
& \ll B^{\frac{4}{3} s-3+\varepsilon} Q^{\frac{3-2 s}{3}} .
\end{aligned}
$$

For the terms $\mathbf{m} \neq \mathbf{0}$ we have $|\mathbf{m}| \leq M \ll R \phi B^{2+\varepsilon}$ as before. So the contribution of the $\mathbf{m}$ with $g^{*}(\mathbf{m}) \neq 0$ can be bound as in (4.16) by

$$
\begin{aligned}
& \ll B^{-\frac{2}{3} s+\varepsilon} \max _{R, \phi} R^{\frac{2}{3}} \phi^{-\frac{2}{3} s+1}\left(R^{\frac{4}{3}-\frac{1}{3} s}+R^{\frac{3}{2}+s} \phi^{2 s} B^{4 s+\varepsilon}\right)^{\frac{2}{3}} \\
& \ll B^{\frac{4}{3} s-3+\varepsilon} Q^{\frac{3-2 s}{3}}+B^{2 s+\varepsilon} \max _{R} R^{\frac{5}{3}+\frac{2}{3} s}(R \hat{Q})^{-\frac{2}{3} s-1} \\
& \ll B^{\frac{4}{3} s-3+\varepsilon} Q^{\frac{3-2 s}{3}}+B^{2 s+\varepsilon} \max _{R} R^{\frac{2}{3}} \hat{Q}^{-\frac{2}{3} s-1} \\
& \ll B^{\frac{4}{3} s-3+\varepsilon} Q^{\frac{3-2 s}{3}}+B^{2 s+\varepsilon} \hat{Q}^{-\frac{2}{3} s-\frac{1}{3}} \\
& \ll B^{\frac{4}{3} s-3+\varepsilon} Q^{\frac{3-2 s}{3}}+B^{\frac{4}{3} s-3+\frac{15-2 s}{6}+\varepsilon},
\end{aligned}
$$

which is satisfactory for $s \geq 8$. 
Finally, the contribution of the $\mathbf{m}$ with $g^{*}(\mathbf{m})=0$ can be bound as in (4.20) by

$$
\begin{aligned}
& \ll B^{-\frac{2}{3} s+\varepsilon} \max _{R, \phi} R^{\frac{14-2 s}{9}} \phi^{-\frac{2}{3} s+1} \\
& \ll B^{-\frac{2}{3} s+\varepsilon}\left(Q B^{-3}\right)^{-\frac{2}{3} s+1} \\
& \ll B^{\frac{4}{3} s-3+\varepsilon} Q^{\frac{3-2 s}{3}},
\end{aligned}
$$

and as (4.21) by

$$
\begin{aligned}
& \ll B^{2 s-4+\varepsilon} \max _{R, \phi} R^{\frac{2}{3} s} \phi^{\frac{2}{3} s-1} \\
& \ll B^{2 s-4+\varepsilon} \max _{R} R^{\frac{2}{3} s}(R \hat{Q})^{-\frac{2}{3} s+1} \\
& \ll B^{2 s-4+\varepsilon} \hat{Q}^{-\frac{2}{3} s+2} \\
& \ll B^{\frac{4}{3} s-3+\frac{6-s}{3}+\varepsilon}
\end{aligned}
$$

and as (4.22) by

$$
\begin{aligned}
& \ll B^{2 s-\frac{16}{9}-\frac{16}{18 s-s}+\varepsilon} \max _{R, \phi} R^{\frac{2}{3} s+\frac{10}{9}-\frac{8}{18 s-9}} \phi^{\frac{2}{3} s+\frac{1}{9}-\frac{8}{18 s-9}} \\
& \ll B^{2 s-\frac{16}{9}-\frac{16}{18 s-s}+\varepsilon} \hat{Q}^{-\frac{2}{3} s-\frac{1}{9}+\frac{8}{18 s-9}} \max _{R} R \\
& \ll B^{\frac{4}{3} s-3-\frac{23-3 s}{9}-\frac{4}{18 s-9}+\varepsilon} \\
& \ll B^{\frac{4}{3} s-3-\frac{1}{9}+\varepsilon} .
\end{aligned}
$$

On choosing $\theta=\frac{19}{892}$, we find that all error terms are $\ll B^{\frac{4}{3} s-3-\frac{19}{4014}+\varepsilon}$ and we can collect the results as

Lemma 4.11. The minor arc contribution is

$$
\int_{\mathfrak{m}}|S(\alpha)|^{\frac{4}{3}} d \alpha \ll B^{\frac{4}{3} s-3-\delta},
$$

where $0<\delta<\frac{19}{4014}$.

\subsection{Conclusion}

Using Lemma 4.10, we can deduce the major arc estimate

$$
\int_{\mathfrak{M}} S(\alpha) \mathrm{e}(-\alpha n) d \alpha \gg B^{s-3-\varepsilon} .
$$

for almost all $n \in \mathcal{A}$, without $p$-adic obstructions. 
Together with the minor arc estimate Lemma 4.11 we can use our Prototype Theorem 2.2 to immediately deduce Theorem 1.2 .

In order to improve further on the number of variables, Hooley [17] and more recently in [18] and [19] makes use of cancellation in the exponential sums when averaged over certain ranges. Unfortunately, inherent in our method, we have to estimate the minor arc integral over the absolute value of the exponential sum, therefore it is not possible to apply results, which depend for example on cancellations occurring in the summation $\sum_{a=1}^{q} S(q, a)$. In order to make use of those results, it seems more promising to work directly on the exponential sum corresponding to the form $f$ and the quadratic polynomial $\mathfrak{q}$, instead of splitting them up, as in the method presented here. However it might be possible to generalize Theorem 1.2 to arbitrary cubic polynomials $f$, where the homogeneous cubic part $f_{0}$ is non-singular, as in [3]. Also, it might be possible to apply the method to cubic forms that split and get corresponding results to the ones by Browning [2]. 


\section{Quartic Forms}

As another application of our Prototype Theorem 2.2, we want to investigate the representation of $n \in \mathcal{A}=\{\mathfrak{q}(y): y \in \mathbb{Z}\} \cap \mathbb{N}$ by absolutely irreducible quartic forms $f \in \mathbb{Z}\left[x_{1}, \ldots, x_{s}\right]$ and prove Theorem 1.3.

\subsection{Preparations}

The general setup for the circle method will be the same as in $[4, \S 8]$ and will be stated briefly. We choose the smooth weight

$$
\omega(t)= \begin{cases}\exp \left(\frac{-1}{1-t^{2}}\right) & \text { for }|t|<1 \\ 0 & \text { otherwise }\end{cases}
$$

and define

$$
\mathrm{w}(\mathbf{x}):=\omega\left(\rho^{-1}\left|\mathbf{x}-\mathbf{x}_{0}\right|\right) \text { and } \mathrm{w}_{B}(\mathbf{x}):=\mathrm{w}\left(\mathbf{x} B^{-1}\right),
$$

as in chapter 4 .

For a small parameter $0<\theta<\frac{1}{5}$, we set $Q=B^{\theta}$ and define the major arcs by

$$
\mathfrak{M}:=\bigcup_{q \leq Q} \bigcup_{a=1}^{q}\left[\frac{a}{q}-B^{-4} Q, \frac{a}{q}+B^{-4} Q\right],
$$

and the corresponding minor arcs as $\mathfrak{m}:=[0,1) \backslash \mathfrak{M}$ modulo 1 .

As in the chapters before, the main task will be to provide the necessary major and minor arc estimates (2.11) and (2.12).

\subsection{Major Arcs}

In order to estimate

$$
\int_{\mathfrak{M}} S(\alpha) \mathrm{e}(-\alpha n) d \alpha=\sum_{q \leq Q} \sum_{a=1}^{q} \int_{|\beta| \leq B^{-4} Q} S(a / q+\beta) \mathrm{e}(-(a / q+\beta) n) d \beta,
$$


we sort the summation over $\mathbf{x}$ occurring in $S(\alpha)$ by residue classes modulo $q$ using the transformation $\mathbf{x} \rightarrow \mathbf{x}+q \mathbf{k}$ as follows.

$$
\begin{aligned}
S(a / q+\beta) & =\sum_{\mathbf{x}(q)} \sum_{\mathbf{k} \in \mathbb{Z}^{s}} \mathrm{e}((a / q+\beta) f(\mathbf{x}+q \mathbf{k})) \mathrm{w}_{B}(\mathbf{x}+q \mathbf{k}) \\
& =\sum_{\mathbf{x}(q)} \mathrm{e}(a f(\mathbf{x}) / q) \sum_{\mathbf{k} \in \mathbb{Z}^{s}} \mathrm{e}(\beta f(\mathbf{x}+q \mathbf{k})) \mathrm{w}_{B}(\mathbf{x}+q \mathbf{k}) .
\end{aligned}
$$

Next, we want to replace the summation over $\mathbf{k} \in \mathbb{Z}^{s}$ by an integration over $\mathbf{t} \in \mathbb{R}^{s}$. Therefore we define $h(\mathbf{k})=\mathrm{e}(\beta f(\mathbf{x}+q \mathbf{k})) \mathrm{w}_{B}(\mathbf{x}+q \mathbf{k})$ and note that for $\mathbf{y} \in[0,1]^{s}$

$$
h(\mathbf{k}+\mathbf{y})=h(\mathbf{k})+\mathrm{O}\left(\max _{\mathbf{y} \in[0,1]^{s}}|\nabla h(\mathbf{k}+\mathbf{y})|\right) .
$$

So the error in replacing the summation by an integration is

$$
\begin{aligned}
\left|\int_{\mathbb{R}^{s}} h(\mathbf{t}) d \mathbf{t}-\sum_{\mathbf{k}_{i} n \mathbb{Z}^{s}} h(\mathbf{k})\right| & \ll \operatorname{meas}(\mathcal{B}) \max _{\mathbf{t} \in \mathcal{B}}|\nabla h(\mathbf{t})| \\
& \ll\left(\frac{B}{q}\right)^{s}\left(q B^{-1}+q|\beta| B^{3}\right) \\
& \ll|\beta| q^{1-s} B^{s+3}+q^{1-s} B^{s-1},
\end{aligned}
$$

where $\mathcal{B}$ is an $s$-dimensional cube with sides of order $\ll q B^{-1}$.

Inserting this into (5.3) and making the change of variables $\mathbf{x}+q \mathbf{k} \rightarrow B \mathbf{t}$, we get

$$
S(a / q+\beta)=B^{s} q^{-s} S(q, a) \int_{\mathbb{R}^{s}} \mathrm{e}\left(\beta B^{4} f(\mathbf{t})\right) \mathrm{w}(\mathbf{t})+\mathrm{O}\left(|\beta| q B^{s+3}+q B^{s-1}\right),
$$

where $S(q, a)=\sum_{\mathbf{x}=1}^{q}{ }^{*} \mathrm{e}(a f(\mathbf{x}) / q)$ and the error term is $\mathrm{O}\left(B^{s-1+2 \theta}\right)$, since $|\beta| \leq B^{-4+\theta}$ and $q \leq B^{\theta}$ on the major arcs.

Hence, the right-hand side of (5.2) becomes

$B^{s} \sum_{q \leq Q} q^{-s} \sum_{a=1}^{q} S(q, a) \mathrm{e}(-a n / q) \int_{|\beta| \leq B^{-4} Q} \int_{\mathbb{R}^{s}} \mathrm{e}\left(\beta\left(B^{4} f(\mathbf{t})-n\right)\right) \mathrm{w}(\mathbf{t}) d \mathbf{t} d \beta+\mathrm{O}\left(B^{s-5+5 \theta}\right)$,

on noting that the major arcs have a measure $\mathrm{O}\left(B^{-4+3 \theta}\right)$ contributing to the error term. 
Finally the transformation $\beta \rightarrow B^{-4} \beta$ leads to

$$
\begin{aligned}
\int_{\mathfrak{M}} S(\alpha) \mathrm{e}(-\alpha n) d \alpha & =B^{s-4} \sum_{q \leq Q} q^{-s} \sum_{a=1}^{q} S(q, a) \mathrm{e}(-a n / q) \int_{|\beta| \leq Q} I(\beta) d \beta+\mathrm{O}\left(B^{n-5+5 \theta}\right) \\
& =B^{s-4} \mathfrak{S}(Q, n) \mathfrak{J}(Q, n)+\mathrm{O}\left(B^{n-5+5 \theta}\right),
\end{aligned}
$$

where

$$
I(\beta)=\int \mathrm{e}\left(\beta\left(f(\mathbf{t})-n B^{-4}\right)\right) \mathrm{w}(\mathbf{t}) d \mathbf{t}
$$

and the the finite versions of the singular series and the singular integral are defined as

$$
\mathfrak{S}(Q, n)=\sum_{q \leq Q} q^{-s} \sum_{a=1}^{q} S(q, a) \mathrm{e}(-a n / q) \quad \text { and } \quad \mathfrak{J}(Q, n)=\int_{|\beta| \leq Q} I(\beta) d \beta
$$

\subsubsection{Singular Series}

To see the absolute convergence $\mathfrak{S}(Q) \rightarrow \mathfrak{S}$ of the singular series

$$
\mathfrak{S}(Q)=\sum_{q \leq Q} q^{-s} \sum_{a=1}^{q} S(q, a) \mathrm{e}(-a n / q)=: \sum_{q \leq Q} A_{n}(q),
$$

we proceed as in section 3.2.1.

Here (3.5) becomes

$$
\begin{aligned}
A_{n}(q) & =q^{-s} \varphi(q)^{-1} \sum_{a=1}^{q} S(q, a) U(q, a n) \\
& \ll q^{-s+\frac{1}{2}+\varepsilon}(q ; n)^{\frac{1}{2}} \max _{(a ; q)=1}|S(q, a)|,
\end{aligned}
$$

using Lemma 3.4.

Now we can write $q=u v$, where $(u ; v)=1$ and $u$ is the square-free part of $q$. This allows us to use [4, Lemma 7], which we state as

Lemma 5.1. For $u \in \mathbb{N}$ square-free and $(u ; a)=1$, we have

$$
S(u, a) \ll u^{(s+\sigma+1) / 2+\varepsilon},
$$

together with $[4$, eq. (6.11)], which we state as 
Lemma 5.2. For arbitrary $q \in \mathbb{N}$ and $(q ; a)=1$, we have

$$
S(q, a) \ll q^{(23 s+\sigma+1) / 24+\varepsilon} .
$$

Since $A_{n}(q)$ is a multiplicative function in $q,(5.5)$ yields

$$
A_{n}(q) \ll q^{-s+\frac{1}{2}+\varepsilon}(q ; n)^{\frac{1}{2}} u^{(s+\sigma+1) / 2+\varepsilon} v^{(23 s+\sigma+1) / 24+\varepsilon} .
$$

So the tail of the singular series can be estimated as

$$
\begin{aligned}
\sum_{q>Q}\left|A_{n}(q)\right| & \ll \sum_{\substack{u v>Q,(u ; v)=1 \\
u \text { square-free } \\
v \text { square-full } \\
\text { ind }}} u^{(-s+\sigma+2) / 2+\varepsilon}(u ; n)^{\frac{1}{2}} v^{(-s+\sigma+13) / 24+\varepsilon}(v ; n)^{\frac{1}{2}} \\
& \ll Q^{-\frac{1}{24}} \sum_{\substack{u v>Q,(u ; v)=1 \\
u \text { square-free } \\
v \text { square-full }}} u^{-2} v^{-\frac{13}{24}}(u v ; n)^{\frac{1}{2}} \rightarrow 0
\end{aligned}
$$

for $s-\sigma \geq 26$. The sum being convergent, since the number of square-full integers $v \leq V$ is $\mathrm{O}\left(V^{\frac{1}{2}}\right)$ and the gcd only contributes $n^{\varepsilon}$ as in (3.6).

With exactly the same arguments that lead to Lemma 3.5 in section 3.2.1, we find that the singular series is $\mathfrak{S}(n) \gg n^{-\varepsilon}$ for almost all $n \leq N$, as long as there are no $p$-adic obstructions.

\subsubsection{Singular Integral}

Browning and Heath-Brown establish the absolute convergence of $\mathfrak{J}(Q, n) \rightarrow \mathfrak{J}\left(B^{-4} n\right)$ for $s-\sigma \geq 26$ and get

$$
\int_{\mathfrak{M}} S(\alpha) \mathrm{e}(-\alpha n) d \alpha=B^{s-4} \mathfrak{S}(n) \mathfrak{J}\left(B^{-4} n\right)+\mathrm{O}\left(B^{s-4-\delta}\right)
$$

for some $\delta>0$ in $[4, \S 10$,Lemma 23]. Note that the additional factor e $(-\alpha n)$, which appears here, does no harm and the analog proof applies to our situation at hand, uniformly in $n$. The arguments that show $\mathfrak{J}\left(B^{-4} n\right) \gg 1$ uniformly in $n \in \tilde{\mathcal{E}}_{N}=[N, \tau N] \cap \mathcal{E}$ in (3.8) are still valid and we can conclude

Lemma 5.3. For $s-\sigma \geq 26$ and almost all $n$ without $p$-adic obstructions, the major arc integral fulfills

$$
\int_{\mathfrak{M}} S(\alpha) \mathrm{e}(-\alpha n) d \alpha \gg B^{s-4-\varepsilon}
$$




\subsection{Minor Arcs}

To treat the minor arc integral (2.12), we apply Dirichlet's approximation theorem and write $\alpha \in \mathfrak{m}$ as $\alpha=a / q+\beta$, where $(a ; q)=1, q \leq \hat{Q}$ and $|\beta| \leq(q \hat{Q})^{-1}$, where $\hat{Q}=B^{\frac{8}{5}+\phi}$ and $\phi>0$.

This is the point, where Browning and Heath-Brown improve on the work of Birch by using van der Corput's method to come up with an alternative bound for $S(a / q+\beta)$. Therefore the range of integration is split up according to the approximation $\alpha=$ $a / q+\beta$ above. Note that $\alpha \in \mathfrak{m}$ implies $q>Q$ or $|\beta|>Q B^{-4}$, which will be indicated by $\star$ in the following integration over $\beta$.

$$
\int_{\mathfrak{m}} S(\alpha) d \alpha=\sum_{q \leq \hat{Q}} \sum_{a=1}^{q} \int_{\beta}^{\star} S(a / q+\beta) d \beta
$$

For $R<q \leq 2 R$ and $t<|\beta| \leq 2 t$ the authors provide bounds $\Upsilon(R, t)$ on

$$
\Sigma(R, t, \pm)=\sum_{R<q \leq 2 R} \sum_{a=1}^{q} \int_{t<|\beta| \leq 2 t}|S(a / q+\beta)| d \beta \ll \sum_{R<q \leq 2 R} R t \Upsilon(R, t) .
$$

Namely [4, Lemma 22], which we slightly reformulate as

Lemma 5.4. We have

$$
\Upsilon(R, t) \ll B^{s+\varepsilon}\left(\hat{Q} B^{-9 / 5}+\mu\right)^{(s-\sigma-1) / 2},
$$

where $\mu=\min \left(\frac{R_{1}^{5 / 7}}{R^{3 / 7}}+R_{1}^{2 / 5} t^{1 / 5} B^{1 / 5}+\frac{R_{1}^{1 / 2}}{B^{1 / 2}}, \frac{R_{1}^{1 / 2} R_{2}^{1 / 4}}{R^{3 / 8}}\right)$ and $R_{1}, R_{2}$ and $R_{3}$ stem from splitting $q=r_{1} r_{2}^{2} r_{3}$ with $r_{1}=\prod_{\substack{p^{e} \| q \\ e \leq 2}} p^{e}, r_{3}=\prod_{\substack{p^{e} \| q \\ e \geq 3,2 \nmid e}} p$ and restricting $r_{i} \sim R_{i}$.

For some ranges of $R$ and $t$ this bound is better than the one obtained by iterated Weyl Differencing [4, Lemma 21], which we state as

Lemma 5.5. For $t>\left(R B^{2}\right)^{-1}$ we have

$$
\Upsilon(R, t) \ll B^{s+\varepsilon}(R t)^{(s-\sigma-1) / 24},
$$

while for $t \leq\left(R B^{2}\right)^{-1}$ we have

$$
\Upsilon(R, t) \ll B^{s-(s-\sigma-1) / 6+\varepsilon}(R t)^{-(s-\sigma-1) / 24} .
$$


It takes quite a lengthy calculation to balance both contributions and get an optimal bound. We do not want to redo the similar calculations for our case, but rather inspect the structure of the argument to see what it produces in out situation.

Note that both Lemma 5.4 and Lemma 5.5 are of the form

$$
\Upsilon(R, t) \ll B^{s+\varepsilon} \Psi(R, t)^{(s-\sigma-1)}
$$

where $\Psi(R, t)$ is such, that

$$
\sum_{R<q \leq 2 R} R t B^{s+\varepsilon} \Psi(R, t)^{(s-\sigma-1)} \ll B^{s-4-\delta},
$$

for all $R$ and $t$ under consideration and some $\delta>0$ as long as $s-\sigma \geq 42$. In other words $\Psi(R, t)$ provides a saving of the form

$$
\sum_{R<q \leq 2 R} R t \Psi(R, t)^{41} \ll B^{-4-\delta}
$$

In our case, we have to estimate

$$
\begin{aligned}
\int_{\mathfrak{m}}|S(\alpha)|^{\frac{4}{3}} d \alpha & =\sum_{q \leq \hat{Q}} \sum_{a=1}^{q} \int_{\beta}^{\star}\left|S\left(\frac{a}{q}+\beta\right)\right|^{\frac{4}{3}} d \beta \\
& \ll B^{\varepsilon} \max _{R \leq \hat{Q}} \sum_{R<q \leq 2 R} \sum_{a=1}^{q} \int_{\beta}^{\star}\left|S\left(\frac{a}{q}+\beta\right)\right|^{\frac{4}{3}} d \beta .
\end{aligned}
$$

Note that there are only $\mathrm{O}(\log B)$ intervals $[R, 2 R]$ up to $\hat{Q}$, so we can restrict the sum over $q$ to one such interval at the cost of $B^{\varepsilon}$.

The ranges $|\beta| \leq B^{-4-\delta} R^{-2}$ are now easily estimated as

$$
\sum_{R<q \leq 2 R} \sum_{a=1}^{q} \int_{|\beta| \leq B^{-4-\delta} R^{-2}}\left|S\left(\frac{a}{q}+\beta\right)\right|^{\frac{4}{3}} d \beta \ll R^{2} B^{-4-\delta} R^{-2} B^{\frac{4}{3} s}=B^{\frac{4}{3} s-4-\delta},
$$

by using the trivial bound $|S(\alpha)| \ll B^{s}$.

The remaining ranges $|\beta|>B^{-4-\delta} R^{-2}$ can be split into $\mathrm{O}(\log B)$ intervals $[t, 2 t]$ at the cost of $B^{\varepsilon}$ and using (5.6) we arrive at 


$$
\begin{aligned}
\sum_{R<q \leq 2 R} \sum_{a=1}^{q} \int_{t<|\beta| \leq 2 t}\left|S\left(\frac{a}{q}+\beta\right)\right|^{\frac{4}{3}} d \beta & \ll \sum_{R<q \leq 2 R} R t \Upsilon(R, t)^{\frac{4}{3}} \\
& \ll B^{\frac{4}{3} s+\varepsilon} \sum_{R<q \leq 2 R} R t \Psi(R, t)^{\frac{4}{3}(s-\sigma-1)} .
\end{aligned}
$$

For $s-\sigma \geq 32$ the sum produces a saving of at least $B^{-4-\delta}$ according to (5.7) and we get

Lemma 5.6. For $s-\sigma \geq 32$ we have the minor arc estimate

$$
\int_{\mathfrak{m}} S(\alpha) d \alpha \ll B^{\frac{4}{3} s-4-\delta+\varepsilon} .
$$

Note that we needed $\frac{4}{3}(s-\sigma-1) \geq 41$, which is equivalent to $s-\sigma \geq 31.75$ instead of $s-\sigma \geq \frac{3}{4} \cdot 42=31.5$. Luckily this small loss does not increase the number of variables needed.

\subsection{Conclusion}

The Prototype Theorem 2.2 together with Lemma 5.3 and Lemma 5.6 complete the proof of Theorem 1.3.

Here the available estimates for the exponential sum $S(\alpha)$ were good enough to carry enough savings through our method to reduce the number of variables needed to represent almost all $n$ to $\frac{3}{4}$ times the number of variables needed for the representation of 0 . This was possible because the provided bounds were somewhat "pointwise" bounds on $|S(a / q+\beta)|$ for $q \sim R$ and $\beta \sim t$. 


\section{Conclusion}

Instead of requiring the explicit major arc and minor arc estimates in Theorem 2.2 it would be nice to reformulate the Prototype-Theorem as

(Desired) Heuristic 6.1. Let $\mathcal{C} \subset \mathbb{Z}\left[x_{1}, \ldots, x_{s}\right]$ be a class of polynomials of degree $d$. If we can prove a theorem, stating that for each $f \in \mathcal{C}$ the equation $f(\mathbf{x})=0$ has non-trivial solutions as soon as $s \geq s_{0}$ by providing an asymptotic formula for the major arc integral of the form

$$
\int_{\mathfrak{M}} S(\alpha) d \alpha=\mathfrak{S} \mathfrak{J} B^{s-d}+\mathrm{O}\left(B^{s-d-\delta}\right)
$$

and a minor arc estimate of the form

$$
\int_{\mathfrak{m}}|S(\alpha)| d \alpha \ll B^{s-d-\delta},
$$

then almost all $n \in \mathcal{A}$ are represented by $f \in \mathcal{C}$ as long as $s \geq \frac{3}{4} s_{0}$ and there are no real or p-adic obstructions.

This would be possible, if the estimates roughly came from estimates for $\alpha \in \mathfrak{m}$ of the form $|S(\alpha)| \ll B^{(1-\gamma) s}$, such that $\gamma s_{0}>d$. As then

$$
|S(\alpha)|^{\frac{4}{3}} \ll B^{\frac{4}{3}(1-\gamma) s}=\mathrm{o}\left(B^{\frac{4}{3} s-d}\right),
$$

for $s>\frac{4}{3} s_{0}$.

But unfortunately the more recent applications of the circle method often do not provide such estimates for the absolute value $|S(\alpha)|$ point-wise, but rather rely on some additional cancellation on averaging over $S(\alpha)$ for certain ranges of $\alpha$. In some cases those estimates are only provided under additional assumptions and geometric arguments are used to find at least one solution to $f(\mathbf{x})=0$ if the assumptions do not hold.

So in practice the Heuristic 6.1 could not be applied directly and some more work has to be done to follow the technical arguments in detail and provide the necessary 
estimates for Theorem 2.2, as worked out in chapter 4 and chapter 5 for the case $d=3$ and $d=4$ respectively.

Finally, one may ask, what happens for arbitrary sets $\mathcal{A} \subset \mathbb{N}$ of size $\left|\mathcal{A}_{N}\right| \sim N^{\frac{1}{k}}$, $k \geq 2$. We want to give a quick heuristic for the number of variables needed by using the method presented before. Therefore we assume that we were able to prove the major arc estimates of our Prototype Theorem 2.2 for some class of polynomials $f \in \mathbb{Z}\left[x_{1}, \ldots, x_{s}\right]$ of degree $\mathrm{d}$. For $\alpha \in \mathfrak{m}$ additionally we assume to have estimates of the form $|S(\alpha)| \ll B^{(1-\gamma) s}$ mentioned above. In this case we get

Heuristic 6.2. If $s_{0}$ is the number of variables needed to prove that $f(\mathbf{x})=0$ has non-trivial solutions under the assumptions above (that is $\gamma s_{0}>d$ ), and $\mathcal{A} \subset \mathbb{N}$ is an arbitrary set of size $\left|\mathcal{A}_{N}\right| \sim N^{\frac{1}{k}}, k \geq 2$, then almost all $n \in \mathcal{A}$ without real or p-adic obstructions are represented by $f$ as soon as $s \geq \frac{2 k-1}{2 k} s_{0}$.

Proof. We proceed as in chapter 2 to get (2.6). Note that the additional factor e $(\alpha n)$ typically gives rather big savings compared to the problem with $n=0$. So the major arc estimates usually should carry through with the help of Lemma 3.4.

As we do not know more about the structure of the set $\mathcal{A}$, we just use the CauchySchwarz inequality and get

$$
\begin{aligned}
\left|\mathcal{E}_{N}\right| & \ll B^{-s+d+\varepsilon} \int_{\mathfrak{m}}|S(\alpha) K(\alpha)| d \alpha \\
& \ll B^{-s+d+\varepsilon}\left(\int_{\mathfrak{m}}|S(\alpha)|^{2} d \alpha\right)^{\frac{1}{2}}\left(\int_{0}^{1}|K(\alpha)|^{2} d \alpha\right)^{\frac{1}{2}} .
\end{aligned}
$$

The integral on the right can be interpreted as counting the number of $n_{1}, n_{2} \in \mathcal{E}_{N}$ with $n_{1}=n_{2}$. Together with the bound on $S(\alpha)$ we have

$$
\left|\mathcal{E}_{N}\right| \ll B^{-s+d+\varepsilon} B^{(1-\gamma) s}\left|\mathcal{E}_{N}\right|^{\frac{1}{2}}
$$

So

$$
\left|\mathcal{E}_{N}\right| \ll B^{2 d-2 \gamma s+\varepsilon} \ll N^{2-\frac{2}{d} \gamma s+\varepsilon} .
$$

In order to prove that this is $\ll N^{\frac{1}{d}-\varepsilon}$, we need $2-\frac{2}{d} \gamma s<\frac{1}{k}$, so $\gamma s>\frac{2 k-1}{2 k} d$, which is fulfilled for $s \geq \frac{2 k-1}{2 k} s_{0}$.

So for sets $\mathcal{A}$ of size $A_{N} \sim N^{\frac{1}{k}}$ our method should need $\frac{2 k-1}{2 k}$ times the number of variables to represent almost all $n \in \mathcal{A}$ without real or $p$-adic obstructions as for the corresponding problem of representing 0 . 
Besides the possible generalizations mentioned at the end of chapter 4.4, another direction of further research might be to reduce the requirement from almost all $n \in \mathcal{A}$ to only some $n \in \mathcal{A}$ and come up with lower bounds for the number of elements in $\mathcal{A}$ that do possess a representation, as done in [8] for instance in the case of sums of five cubes. This might lead to insights to representable sets for forms in fewer variables than needed for the representation of almost all $n \in \mathcal{A}$. 


\section{Bibliography}

[1] B. J. Birch. Forms in many variables. Proc. Roy. Soc. Ser. A, 265:245-263, $1961 / 1962$.

[2] T. D. Browning. Rational points on cubic hypersurfaces that split off a form. Compos. Math., 146(4):853-885, 2010. With an appendix by J.-L. ColliotThélène.

[3] T. D. Browning and D. R. Heath-Brown. Integral points on cubic hypersurfaces. In Analytic number theory, pages 75-90. Cambridge Univ. Press, Cambridge, 2009 .

[4] T. D. Browning and D. R. Heath-Brown. Rational points on quartic hypersurfaces. J. Reine Angew. Math., 629:37-88, 2009.

[5] J. Brüdern, K. Kawada, and T. D. Wooley. Additive representation in thin sequences. I. Waring's problem for cubes. Ann. Sci. École Norm. Sup. (4), 34(4):471-501, 2001.

[6] J. Brüdern, K. Kawada, and T. D. Wooley. Additive representation in thin sequences. II. The binary Goldbach problem. Mathematika, 47(1-2):117-125 (2002), 2000.

[7] J. Brüdern, K. Kawada, and T. D. Wooley. Additive representation in thin sequences. III. Asymptotic formulae. Acta Arith., 100(3):267-289, 2001.

[8] J. Brüdern, K. Kawada, and T. D. Wooley. Additive representation in thin sequences. IV. Lower bound methods. Q. J. Math., 52(4):423-436, 2001.

[9] J. Brüdern, K. Kawada, and T. D. Wooley. Additive representation in thin sequences. V. Mixed problems of Waring's type. Math. Scand., 92(2):181-209, 2003.

[10] J. Brüdern, K. Kawada, and T. D. Wooley. Additive representation in thin sequences. VI. Representing primes, and related problems. Glasg. Math. J., 44(3):419-434, 2002.

[11] J. Brüdern, K. Kawada, and T. D. Wooley. Additive representation in thin sequences. VII. Restricted moments of the number of representations. Tsukuba J. Math., 32(2):383-406, 2008. 
[12] J. Brüdern, K. Kawada, and T. D. Wooley. Additive representation in thin sequences, VIII: Diophantine inequalities in review. In Number theory, volume 6 of Ser. Number Theory Appl., pages 20-79. World Sci. Publ., Hackensack, NJ, 2010 .

[13] H. Davenport. Analytic methods for Diophantine equations and Diophantine inequalities, volume 1962 of The University of Michigan, Fall Semester. Ann Arbor Publishers, Ann Arbor, Mich., 1963.

[14] P. Deligne. La conjecture de Weil. I. Inst. Hautes Études Sci. Publ. Math., (43):273-307, 1974.

[15] D. R. Heath-Brown. Cubic forms in ten variables. Proc. London Math. Soc. (3), 47(2):225-257, 1983.

[16] D. Hilbert. Beweis für die Darstellbarkeit der ganzen Zahlen durch eine feste Anzahl $n^{\text {ter }}$ Potenzen (Waringsches Problem). Math. Ann., 67(3):281-300, 1909.

[17] C. Hooley. On nonary cubic forms. J. Reine Angew. Math., 386:32-98, 1988.

[18] C. Hooley. On octonary cubic forms. Proc. Lond. Math. Soc. (3), 109(1):241-281, 2014.

[19] C. Hooley. On octonary cubic forms: II. Bull. Lond. Math. Soc., 47(1):85-94, 2015.

[20] L.-K. Hua. On the representation of numbers as the sums of the powers of primes. Math. Z., 44(1):335-346, 1939. 




\section{Danksagung}

An dieser Stelle möchte ich mich bei all denjenigen bedanken, die mich während des Studiums und meiner Promotion unterstützt haben. Dabei geht mein besonderer Dank an Herrn Professor Dr. Jörg Brüdern, den Betreuer meiner Doktorarbeit, der mir stets mit neuen Blickwinkeln und hilfreichen Anregungen zur Seite stand, falls sich ein scheinbar unüberwindbares Hindernis aufzubauen drohte. Die gemeinsame Betreuung etlicher Lehrveranstaltungen während der Promotionszeit wird mir stets in positiver Erinnerung bleiben.

Außerdem geht ein spezieller Gruß an meine Kommilitonen, durch die die Studienzeit nie langweilig wurde und zu denen - trotz mittlerweile größerer geographischer Entfernung - immernoch enger Kontakt besteht. Darunter aus der Zeit in Göttingen vor allem Sören und Deniz, sowie seit Studienanfangszeit Markus, mit dem ich zusammen schon über so mancher Aufgabe gegrübelt habe.

Nicht zuletzt möchte ich meinen Eltern danken, die mir das Studium ermögicht und mich stets darin unterstützt haben. 